\title{
Functional annotation of human long noncoding RNAs via molecular phenotyping
}

Jordan A. Ramilowski, 1,2,47 Chi Wai Yip, 1,2,47 Saumya Agrawal, ${ }^{1,2}$ Jen-Chien Chang, 1,2 Yari Ciani, ${ }^{3}$ Ivan V. Kulakovskiy, ${ }^{4,5}$ Mickaël Mendez, ${ }^{6}$ Jasmine Li Ching Ooi, ${ }^{2}$ John F. Ouyang, ${ }^{7}$ Nick Parkinson, ${ }^{8}$ Andreas Petri, ${ }^{9}$ Leonie Roos, ${ }^{10,11}$ Jessica Severin, ${ }^{1,2}$ Kayoko Yasuzawa, ${ }^{1,2}$ Imad Abugessaisa, ${ }^{1,2}$ Altuna Akalin, ${ }^{12}$ Ivan V. Antonov, ${ }^{13}$ Erik Arner, ${ }^{1,2}$ Alessandro Bonetti, ${ }^{2}$ Hidemasa Bono, ${ }^{14}$ Beatrice Borsari, ${ }^{15}$

Frank Brombacher, ${ }^{16,17}$ Christopher J.F. Cameron, ${ }^{18,23,46}$ Carlo Vittorio Cannistraci, ${ }^{19,20}$ Ryan Cardenas, ${ }^{21}$ Melissa Cardon, ${ }^{1}$ Howard Chang, ${ }^{22}$ Josée Dostie, $^{23}$ Luca Ducoli, $^{24}$ Alexander Favorov, ${ }^{25,26}$ Alexandre Fort, ${ }^{2}$ Diego Garrido, ${ }^{15}$ Noa Gil, ${ }^{27}$ Juliette Gimenez, ${ }^{28}$ Reto Guler, ${ }^{16,17}$ Lusy Handoko, ${ }^{2}$ Jayson Harshbarger, ${ }^{2}$ Akira Hasegawa, ${ }^{1,2}$ Yuki Hasegawa, ${ }^{2}$ Kosuke Hashimoto, ${ }^{1,2}$ Norihito Hayatsu, ${ }^{1}$ Peter Heutink, ${ }^{29}$ Tetsuro Hirose, $^{30}$ Eddie L. Imada, ${ }^{26}$ Masayoshi Itoh, ${ }^{2,31}$

Bogumil Kaczkowski, ${ }^{1,2}$ Aditi Kanhere, ${ }^{21}$ Emily Kawabata, ${ }^{2}$ Hideya Kawaji, ${ }^{31}$ Tsugumi Kawashima, ${ }^{1,2}$ S. Thomas Kelly, ${ }^{1}$ Miki Kojima, ${ }^{1,2}$ Naoto Kondo, ${ }^{2}$ Haruhiko Koseki, ${ }^{1}$ Tsukasa Kouno, ${ }^{1,2}$ Anton Kratz, ${ }^{2}$ Mariola Kurowska-Stolarska, ${ }^{32}$ Andrew Tae Jun Kwon,, ${ }^{1,2}$ Jeffrey Leek, ${ }^{26}$ Andreas Lennartsson, ${ }^{33}$ Marina Lizio, 1,2

Fernando López-Redondo, 1,2 Joachim Luginbühl, 1,2 Shiori Maeda, ${ }^{1}$

Vsevolod J. Makeev, ${ }^{25,34}$ Luigi Marchionni, ${ }^{26}$ Yulia A. Medvedeva, ${ }^{13,34}$ Aki Minoda, ${ }^{1,2}$ Ferenc Müller, ${ }^{21}$ Manuel Muñoz-Aguirre, ${ }^{15}$ Mitsuyoshi Murata, ${ }^{1,2}$ Hiromi Nishiyori, ${ }^{1,2}$ Kazuhiro R. Nitta, ${ }^{1,2}$ Shuhei Noguchi, ${ }^{1,2}$ Yukihiko Noro, ${ }^{2}$ Ramil Nurtdinov, ${ }^{15}$ Yasushi Okazaki, ${ }^{1,2}$ Valerio Orlando, ${ }^{35}$ Denis Paquette, ${ }^{23}$ Callum J.C. Parr, ${ }^{1}$ Owen J.L. Rackham, ${ }^{7}$ Patrizia Rizzu, ${ }^{29}$ Diego Fernando Sánchez Martinez, ${ }^{26}$ Albin Sandelin, ${ }^{36}$ Pillay Sanjana, ${ }^{21}$ Colin A.M. Semple, ${ }^{37}$ Youtaro Shibayama, $^{1,2}$ Divya M. Sivaraman, 1,2 Takahiro Suzuki, ${ }^{1,2}$ Suzannah C. Szumowski, ${ }^{2}$ Michihira Tagami, ${ }^{1,2}$ Martin S. Taylor, ${ }^{37}$ Chikashi Terao, ${ }^{1}$ Malte Thodberg, ${ }^{36}$ Supat Thongjuea, ${ }^{2}$ Vidisha Tripathi, ${ }^{38}$ Igor Ulitsky, ${ }^{27}$ Roberto Verardo, ${ }^{3}$ Ilya E. Vorontsov, ${ }^{25}$ Chinatsu Yamamoto, ${ }^{2}$ Robert S. Young, ${ }^{39}$ J. Kenneth Baillie, ${ }^{8}$ Alistair R.R. Forrest, 1,2,40 Roderic Guigó, ${ }^{15,41}$ Michael M. Hoffman, 42 Chung Chau Hon, 1,2 Takeya Kasukawa, ${ }^{1,2}$ Sakari Kauppinen, ${ }^{9}$ Juha Kere, ${ }^{33,43}$ Boris Lenhard, ${ }^{10,11,44}$ Claudio Schneider, ${ }^{3,45}$ Harukazu Suzuki, 1,2 Ken Yagi, 1,2 Michiel J.L. de Hoon, ${ }^{1,2}$ Jay W. Shin, 1,2 and Piero Carninci,2

\footnotetext{
${ }^{47}$ These authors contributed equally to this work.

Corresponding authors: michiel.dehoon@riken.jp, jay.shin@riken.jp, carninci@riken.jp

Article published online before print. Article, supplemental material, and publication date are at http://www.genome.org/cgi/doi/10.1101/gr.254219.119.

Freely available online through the Genome Research Open Access option.
}

(C) 2020 Ramilowski et al. This article, published in Genome Research, is available under a Creative Commons License (Attribution 4.0 International), as described at http://creativecommons.org/licenses/by/4.0/. 
${ }^{1}$ RIKEN Center for Integrative Medical Sciences, Yokohama, Kanagawa 230-0045, Japan; ${ }^{2}$ RIKEN Center for Life Science Technologies, Yokohama, Kanagawa 230-0045, Japan; ${ }^{3}$ Laboratorio Nazionale Consorzio Interuniversitario Biotecnologie (CIB), Trieste 34127, Italy; ${ }^{4}$ Engelhardt Institute of Molecular Biology, Russian Academy of Sciences, Moscow 119991, Russia; ${ }^{5}$ Institute of Protein Research, Russian Academy of Sciences, Pushchino 142290, Russia; ${ }^{6}$ Department of Computer Science, University of Toronto, Toronto, Ontario M5S 1A1, Canada; ${ }^{7}$ Program in Cardiovascular and Metabolic Disorders, Duke-National University of Singapore Medical School, Singapore 169857, Singapore; ${ }^{8}$ Roslin Institute, University of Edinburgh, Edinburgh EH25 9RG, United Kingdom; ${ }^{9}$ Center for RNA Medicine, Department of Clinical Medicine, Aalborg University, Copenhagen 9220, Denmark; ${ }^{10}$ Institute of Clinical Sciences, Faculty of Medicine, Imperial College London, London W12 0NN, United Kingdom; ${ }^{11}$ Computational Regulatory Genomics, MRC London Institute of Medical Sciences, London W12 ONN, United Kingdom; ${ }^{12}$ Berlin Institute for Medical Systems Biology, Max Delbrük Center for Molecular Medicine in the Helmholtz Association, Berlin 13125, Germany; ${ }^{13}$ Institute of Bioengineering, Research Center of Biotechnology, Russian Academy of Sciences, Moscow 117312, Russia; ${ }^{14}$ Graduate School of Integrated Sciences for Life, Hiroshima University, Higashi-Hiroshima City 739-0046, Japan; ${ }^{15}$ Centre for Genomic Regulation (CRG), The Barcelona Institute of Science and Technology, Barcelona, Catalonia 08003, Spain; ${ }^{16}$ International Centre for Genetic Engineering and Biotechnology (ICGEB), University of Cape Town, Cape Town 7925, South Africa; ${ }^{17}$ Institute of Infectious Diseases and Molecular Medicine (IDM), Department of Pathology, Division of Immunology and South African Medical Research Council (SAMRC) Immunology of Infectious Diseases, Faculty of Health Sciences, University of Cape Town, Cape Town 7925, South Africa; ${ }^{18}$ School of Computer Science, McGill University, Montréal, Québec H3G 1Y6, Canada; ${ }^{19}$ Biomedical Cybernetics Group, Biotechnology Center (BIOTEC), Center for Molecular and Cellular Bioengineering (CMCB), Center for Systems Biology Dresden (CSBD), Cluster of Excellence Physics of Life (PoL), Department of Physics, Technische Universität Dresden, Dresden 01062, Germany; ${ }^{20}$ Center for Complex Network Intelligence (CCNI) at the Tsinghua Laboratory of Brain and Intelligence (THBI), Department of Bioengineering, Tsinghua University, Beijing 100084, China; ${ }^{21}$ Institute of Cancer and Genomic Sciences, College of Medical and Dental Sciences, University of Birmingham, Birmingham B15 2TT, United Kingdom; ${ }^{22}$ Center for Personal Dynamic Regulome, Stanford University, Stanford, California 94305, USA; ${ }^{23}$ Department of Biochemistry, Rosalind and Morris Goodman Cancer Research Center, McGill University, Montréal, Québec H3G 1Y6, Canada; ${ }^{24}$ Institute of Pharmaceutical Sciences, Swiss Federal Institute of Technology, Zurich 8093, Switzerland; ${ }^{25}$ Department of Computational Systems Biology, Vavilov Institute of General Genetics, Russian Academy of Sciences, Moscow 119991, Russia; ${ }^{26}$ Department of Oncology, Johns Hopkins University, Baltimore, Maryland 21 287, USA; ${ }^{27}$ Department of Biological Regulation, Weizmann Institute of Science, Rehovot 76100, Israel; ${ }^{28}$ Epigenetics and Genome Reprogramming Laboratory, IRCCS Fondazione Santa Lucia, Rome 00179, Italy; ${ }^{29}$ Genome Biology of Neurodegenerative Diseases, German Center for Neurodegenerative Diseases (DZNE), Tübingen 72076, Germany; ${ }^{30}$ Graduate School of Frontier Biosciences, Osaka University, Suita 565-0871, Japan; ${ }^{31}$ RIKEN Preventive Medicine and Diagnosis Innovation Program (PMI), Saitama 351-01 98, Japan; ${ }^{32}$ Institute of Infection, Immunity, and Inflammation, University of Glasgow, Glasgow, Scotland G12 8QQ, United Kingdom; ${ }^{33}$ Department of Biosciences and Nutrition, Karolinska Institutet, Huddinge 14157, Sweden; ${ }^{34}$ Moscow Institute of Physics and Technology, Dolgoprudny 141701, Russia; ${ }^{35}$ Biological and Environmental Sciences and Engineering Division, King Abdullah University of Science and Technology, Thuwal 23955-6900, Kingdom of Saudi Arabia; ${ }^{36}$ Department of Biology and BRIC, University of Copenhagen, Denmark, Copenhagen N DK2200, Denmark; ${ }^{37}$ MRC Human Genetics Unit, University of Edinburgh, Edinburgh EH4 2XU, United Kingdom; ${ }^{38}$ National Centre for Cell Science, Pune, Maharashtra 411007, India; ${ }^{39}$ Centre for Global Health Research, Usher Institute, University of Edinburgh, Edinburgh EH8 9AG, United Kingdom; ${ }^{40}$ Harry Perkins Institute of Medical Research, QEII Medical Centre and Centre for Medical Research, The University of Western Australia, Nedlands, Perth, Western Australia 6009, Australia; ${ }^{41}$ Universitat Pompeu Fabra (UPF), Barcelona, Catalonia 08002, Spain; ${ }^{42}$ Princess Margaret Cancer Centre, Toronto, Ontario M5G 1L7, Canada; ${ }^{43}$ Stem Cells and Metabolism Research Program, University of Helsinki and Folkhälsan Research Center, 00290 Helsinki, Finland;

${ }^{44}$ Sars International Centre for Marine Molecular Biology, University of Bergen, Bergen N-5008, Norway; ${ }^{45}$ Department of Medicine and Consorzio Interuniversitario Biotecnologie p.zle Kolbe 1 University of Udine, Udine 33100, Italy; ${ }^{46}$ Department of Molecular Biophysics and Biochemistry, Yale University, New Haven, Connecticut 06510, USA

Long noncoding RNAs (IncRNAs) constitute the majority of transcripts in the mammalian genomes, and yet, their functions remain largely unknown. As part of the FANTOM6 project, we systematically knocked down the expression of 285 IncRNAs in human dermal fibroblasts and quantified cellular growth, morphological changes, and transcriptomic responses using Capped Analysis of Gene Expression (CAGE). Antisense oligonucleotides targeting the same IncRNAs exhibited global concordance, and the molecular phenotype, measured by CAGE, recapitulated the observed cellular phenotypes while providing additional insights on the affected genes and pathways. Here, we disseminate the largest-todate IncRNA knockdown data set with molecular phenotyping (over 1000 CAGE deep-sequencing libraries) for further exploration and highlight functional roles for ZNF213-ASI and Inc-KHDC3L-2.

[Supplemental material is available for this article.] 
Over 50,000 loci in the human genome transcribe long noncoding RNAs (lncRNAs) (Iyer et al. 2015; Hon et al. 2017), which are defined as transcripts at least 200 nucleotides (nt) long with low or no protein-coding potential. Although lncRNA genes outnumber protein-coding genes in mammalian genomes, they are comparatively less conserved (Ulitsky 2016), lowly expressed, and more cell-type-specific (Hon et al. 2017). However, the evolutionary conservation of lncRNA promoters (Carninci et al. 2005) and the structural motifs of IncRNAs (Chu et al. 2015; Xue et al. 2016) suggest that IncRNAs are fundamental biological regulators. To date, only a few hundred human IncRNAs have been extensively characterized (de Hoon et al. 2015; Quek et al. 2015; Volders et al. 2015; Ma et al. 2019), revealing their roles in regulating transcription (Engreitz et al. 2016b), translation (Carrieri et al. 2012), and chromatin state (Gupta et al. 2010; Guttman et al. 2011; Guttman and Rinn 2012; Quinn and Chang 2016; Ransohoff et al. 2018).

Our recent FANTOM5 computational analysis showed that 19,175 (out of 27,919 ) human lncRNA loci are functionally implicated (Hon et al. 2017). Yet, genomic screens are necessary to comprehensively characterize each lncRNA. One common approach of gene knockdown followed by a cellular phenotype assay typically characterizes a small percentage of lncRNAs for a single observable phenotype. For example, a recent large-scale screening using CRISPR interference (CRISPRi) found that $\sim 3.7 \%$ of targeted IncRNA loci are essential for cell growth or viability in a cell-typespecific manner (Liu et al. 2017). In addition, CRISPR-Cas9 experiments targeting splice sites identified $\sim 2.1 \%$ of lncRNAs that affect growth of K562 (Liu et al. 2018), and a CRISPR activation study revealed $\sim 0.11 \%$ lncRNAs to be important for drug resistance in melanoma (Joung et al. 2017). However, many of these studies target the genomic DNA, potentially perturbing the chromatin architecture, or focus on a single cellular assay, possibly missing other relevant functions and underlying molecular pathways.

As a part of the FANTOM6 pilot project, we established an automated high-throughput cell culture platform to suppress 285 IncRNAs expressed in human primary dermal fibroblasts (HDFs) using antisense LNA-modified GapmeR antisense oligonucleotide (ASO) technology (Roux et al. 2017). We then quantified the effect of each knockdown on cell growth and morphology using realtime imaging, followed by Cap Analysis Gene Expression (CAGE) (Murata et al. 2014) deep sequencing to reveal molecular pathways associated with each IncRNA. In contrast to cellular phenotyping, molecular phenotyping provides a detailed assessment of the response to a lncRNA knockdown at the molecular level, allowing biological pathways to be associated to lncRNAs even in the absence of an observable cellular phenotype. All data and analysis results are publicly available (see Data access), and results can be interactively explored using our in-house portal (https://fantom.gsc.riken .jp/zenbu/reports/\#FANTOM6).

\section{Results}

\section{Selection and ASO-mediated knockdown of IncRNA targets}

Human dermal fibroblasts are nontransformed primary cells that are commonly used for investigating cellular reprogramming (Takahashi et al. 2007; Ambasudhan et al. 2011), wound healing (Li and Wang 2011), fibrosis (Kendall and Feghali-Bostwick 2014), and cancer (Kalluri 2016). Here, an unbiased selection of IncRNAs expressed in HDFs was performed to choose 285 lncRNAs for functional interrogation (Methods; Supplemental
Table S1; Fig. 1A-C). Using RNA-seq profiling of fractionated RNA, we annotated the IncRNA subcellular localization as the chromatin-bound (35\%), nucleus-soluble (27\%), or cytoplasmic (38\%) (Fig. 1D). We then designed a minimum of five non-overlapping antisense oligonucleotides against each lncRNA (Supplemental Methods; Supplemental Table S2; Fig. 1E,F) and transfected them individually using an automated cell culture platform to minimize experimental variability (Fig. 1G). The overall knockdown efficiencies across 2021 ASOs resulted in median value of $45.4 \%$, and we could successfully knockdown 879 out of 2021 (43.5\%) ASOs (>40\% knockdown efficiency in at least two primer pairs or $>60 \%$ in one primer pair) (Supplemental Table S2). ASOs targeting exons or introns were equally effective, and knockdown efficiencies were independent of the genomic class, expression level, and subcellular localization of the lncRNA (Supplemental Fig. S1A-D).

\section{A subset of IncRNAs are associated with cell growth and morphology changes}

To evaluate the effect of each lncRNA knockdown on cell growth and morphology, we imaged ASO-transfected HDFs in duplicate every $3 \mathrm{~h}$ for a total of $48 \mathrm{~h}$ (Supplemental Table S3) and estimated their growth rate based on cell confluence measurements (Fig. 2A,B). First, we observed across all ASOs that changes in cell growth and morphological parameters were significantly correlated with knockdown efficiency (Supplemental Fig. S1E). Considering both successful knockdown and significant growth inhibition (Student's two-sided $t$-test FDR $\leq 0.05), 246$ out of 879 ASOs ( 28\%) showed cellular phenotype (Fig. 2C; Supplemental Table S3).

To assess globally whether the observed growth inhibition is IncRNA-specific, we used all 194 lncRNAs successfully targeted by at least two ASOs (Supplemental Fig. S2A) and found that ASOs targeting the same lncRNA were significantly more likely to have a concordant growth response than ASOs targeting different lncRNA (empirical $P=0.00037$ ) (Supplemental Methods; Supplemental Fig. S2B). However, different ASOs targeting the same IncRNA typically showed different effects on growth, possibly due to variable knockdown efficiencies or differences in targeted lncRNA isoforms, as well as off-target effects. To reliably identify target-specific cellular phenotype, we applied conditional cutoffs based on the number of successful ASOs per each IncRNA (Supplemental Methods; Supplemental Fig. S2C) and identified 15/194 lncRNAs $(7.7 \%)$ with growth phenotype (adjusted background $<5 \%$ ) (Supplemental Fig. S2D). We validated $A 1 B G-A S 1$, which was previously implicated in cell growth (Bai et al. 2019), CATG00000089639, RP11-195F19.9, and ZNF213-AS1 by measuring the MKI67 proliferation protein marker upon knockdown with siRNAs and with selected ASOs (Fig. 2D; Supplemental Fig. S2E).

In addition to cell growth, we also explored changes in cell morphology (Fig. 2E). Using a machine learning-assisted workflow (Methods), each cell was segmented and its morphological features representing various aspects of cell shapes and sizes were quantified (Fig. 2F; Supplemental Table S3; Carpenter et al. 2006). As an example, knockdown of 14/194 lncRNAs (7.2\%) affected the spindle-like morphology of fibroblasts, as indicated by a consistent decrease in their observed eccentricity without reducing the cell number, suggesting possible cellular transformation toward epithelial-like states. Collectively, we observed 59/194 IncRNAs ( 30\%) affecting cell growth and/or morphological parameters (Fig. 2G; Supplemental Table S3).

\section{Genome Research}

www.genome.org 
A

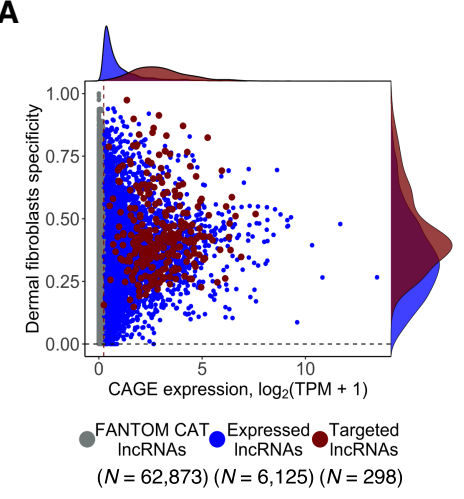

D

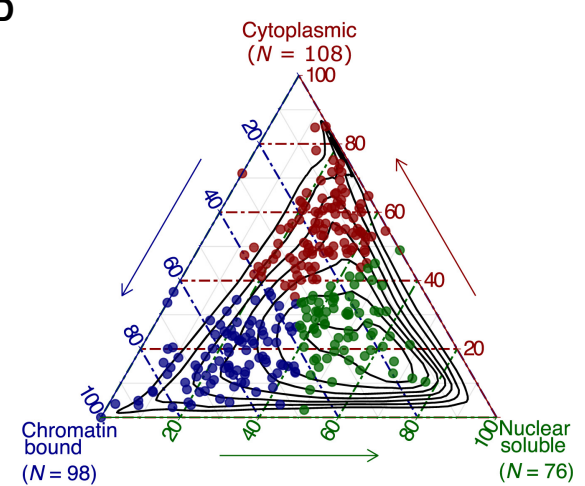

$\mathbf{F}$

\begin{tabular}{l|cccc} 
& $\begin{array}{c}\text { All } \\
\text { Targets }\end{array}$ & $\begin{array}{c}\text { All } \\
\text { ASOs }\end{array}$ & $\begin{array}{c}\text { CAGE } \\
\text { Targets }\end{array}$ & $\begin{array}{c}\text { CAGE } \\
\text { ASOs }\end{array}$ \\
\hline Targeted IncRNAs & 285 & 2,055 & 154 & 340 \\
Positive Controls & 18 & 97 & 13 & 28 \\
Negative Controls & 2 & 2 & 2 & 2 \\
*MALAT1 & 1 & 1 & 1 & 1 \\
\hline
\end{tabular}

*Experimental Control
B

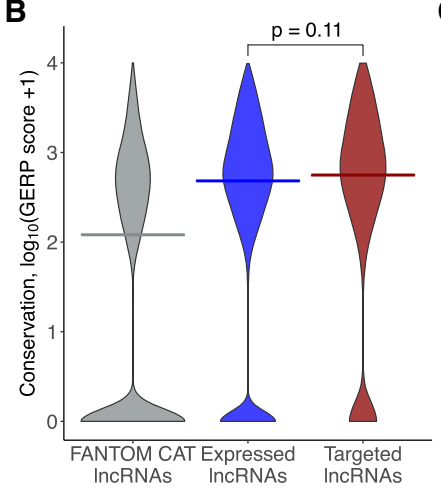

C

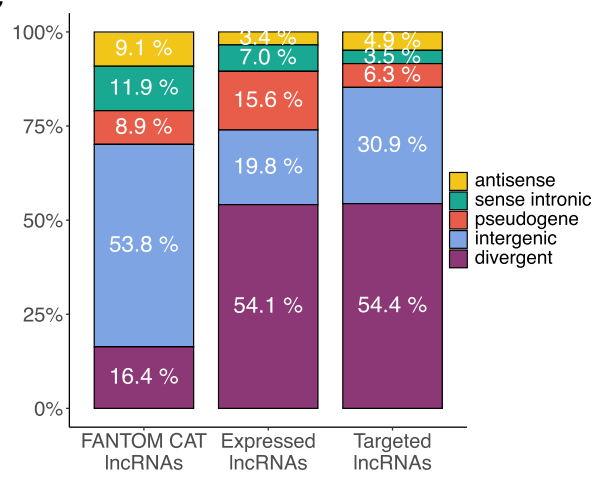

E

Chr $163,135,000-3,128,500$ (ZNF213-AS1)
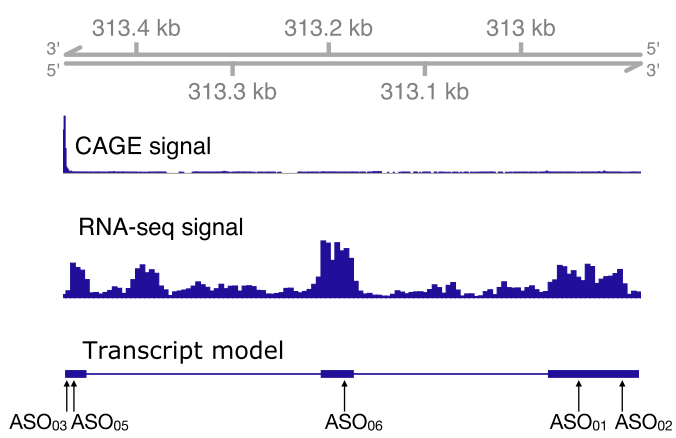

G

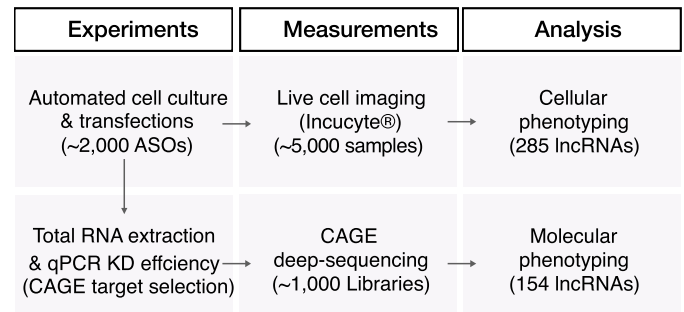

Figure 1. Selection of IncRNA targets, their properties, and the study overview. (A) CAGE expression levels at $\log _{2}$ TPM (tags per million) and human dermal fibroblasts (HDFs) specificity of IncRNAs in the FANTOM CAT catalog (Hon et al. 2017) ( $N=62,873$; gray), IncRNAs expressed in HDFs ( $N=6125$; blue), and targeted IncRNAs ( $N=285$; red). The dashed vertical line indicates most lowly expressed IncRNA target $(\sim 0.2$ TPM). ( $B$ ) Gene conservation levels of IncRNAs in the FANTOM CAT catalog (gray), IncRNAs expressed in HDFs (blue), and targeted IncRNAs (red). Crossbars indicate the median. No significant difference is observed when comparing targeted and expressed in HDF IncRNAs (Wilcoxon $P=0.11$ ). (C) Similar to that in $B$ but for genomic classes of IncRNAs. Most of the targeted IncRNAs and those expressed in HDFs are expressed from divergent promoters. $(D)$ Subcellular localization (based on relative abundances from RNA-seq fractionation data) for targeted IncRNAs. Chromatin-bound ( $N=98$; blue); nuclear soluble ( $N=76$; green); cytoplasmic ( $N=108$; red). Black contours represent the distribution of all InCRNAs expressed in HDFs. (E) Example of ZNF213-AS1 loci showing transcript model, CAGE, and RNA-seq signal along with targeting ASOs. $(F)$ Number of ASOs for target IncRNAs and controls used in the experiment. $(G)$ Schematics of the study.

\section{Molecular phenotyping by CAGE recapitulates cellular phenotypes and highlights functions of IncRNAs}

Next, we selected 340 ASOs with high knockdown efficiencies (mostly $>50 \%$; median $71.4 \%$ ) and sequenced 970 CAGE libraries to analyze 154 IncRNAs (Fig. 3A; Supplemental Table S4). To assess functional implications by individual ASOs, we performed differential gene expression, Motif Activity Response Analysis (MARA) (The FANTOM Consortium et al. 2009), and Gene Set Enrichment Analysis (GSEA) (Fig. 3B-F; Subramanian et al. 2005), and compared them with cellular phenotype.

We globally observed significant knockdown-mediated transcriptomic changes (which generally correlated with KD efficiency)
(Supplemental Fig. S3A), with $\sim 57 \%$ of ASOs showing at least 10 differentially expressed genes (FDR $\leq 0.05$; abs $\left[\log _{2} \mathrm{FC}\right]>0.5$ ). For 84 divergent-antisense IncRNAs (targeted by 186 independent ASOs) (Supplemental Methods), we found their partner gene to be generally unchanged (median $\operatorname{abs}\left[\log _{2} \mathrm{FC}\right]=\sim 0.13$ ), with an exception of two significantly down-regulated and three significantly up-regulated genes (FDR $\leq 0.05$ ) (Supplemental Fig. S3B). We have, however, noticed a common response in a large number of ASOs ( $30 \%-$ $35 \%$ of all responding ASOs), such as down-regulation of cellcycle-related pathways, up-regulated stress genes and pathways, or altered cell metabolism and energetics (Supplemental Fig. S3C,D).

When comparing knockdown-mediated molecular and cellular response, we found that transcription factor motifs that 
A

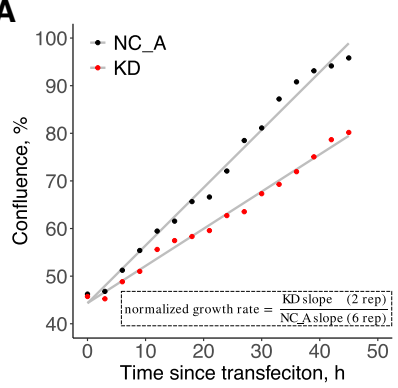

D

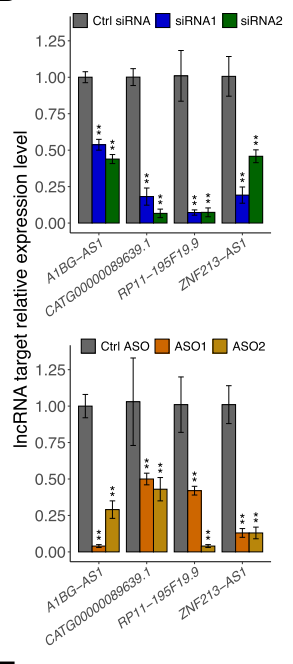

E LINC00630(ASO_02)

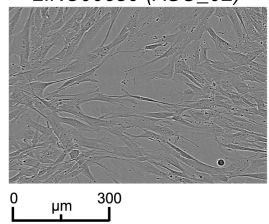

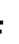

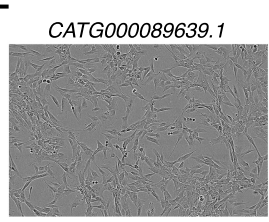

Raw Incucyte image
B

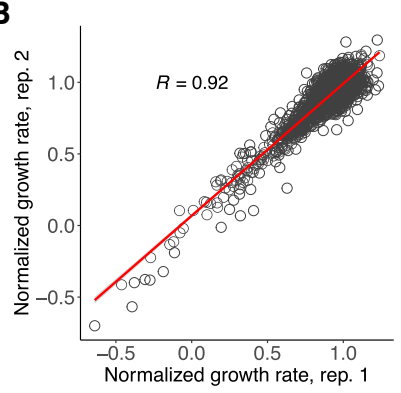

C

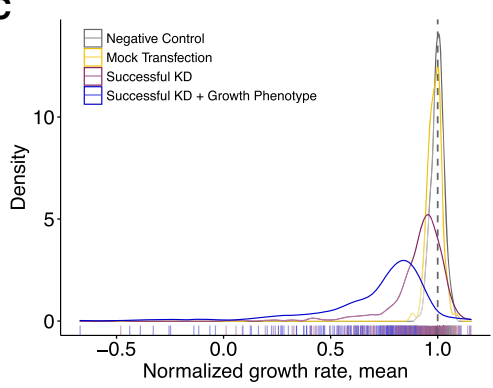

G

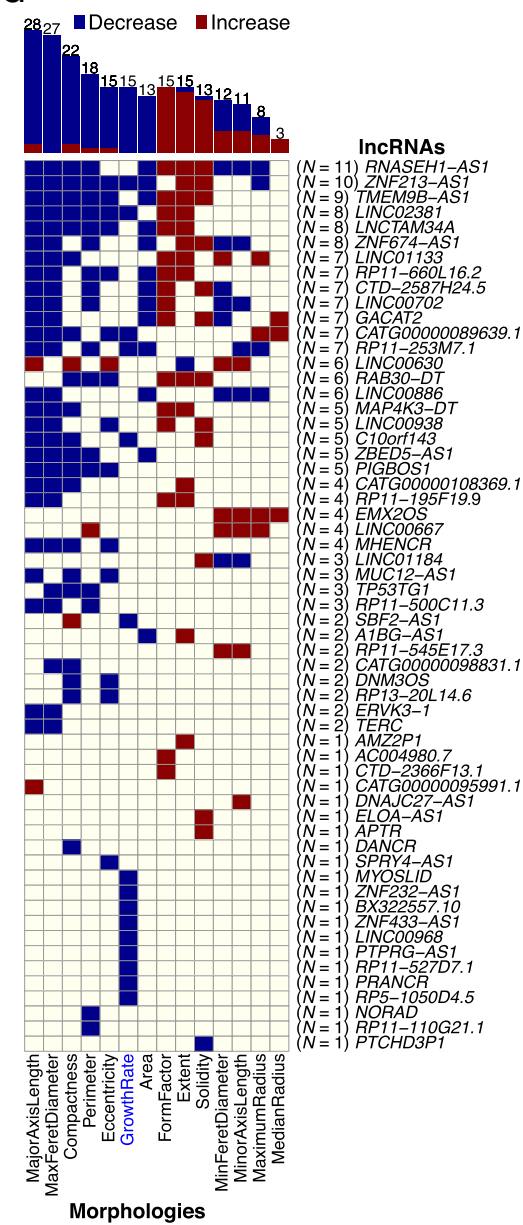

Figure 2. Cell growth and morphology assessment. (A) Selected example (PTPRG1-AS1) showing the normalized growth rate estimation using a matching NC_A (negative control). (B) Correlation of the normalized growth rate for technical duplicates across 2456 Incucyte samples. (C) Density distribution of normalized growth rates (technical replicates averaged) 252 ASOs targeting IncRNAs with successful knockdown (KD) and growth phenotype (blue) consistent in two replicates (FDR $<0.05$ as compared to matching NC_A; 246 ASOs inhibited growth), 627 ASOs targeting IncRNAs with successful KD (purple), 270 negative control (NC_A) samples (gray), and 90 mock-transfected cells (Lipofectamine only) samples (yellow). (D) MKI67 staining (growth inhibition validation) for four selected IncRNA targets after siRNA and ASOs suppression. (E) Incucyte cell images of selected distinct cell morphologies changes upon an IncRNA KD. (F) An overview of the cell morphology imaging processing pipeline using a novel IncRNA target, CATC000089639.1, as an example. $(G)$ IncRNAs $(N=59)$ significantly $(F D R<0.05)$ and consistently (after adjusting for the number of successfully targeting ASOs) affecting cell growth $(N=15)$ and cell morphologies $(N=44)$.

promote cell growth, including TFDP1, E2F1,2,3, and EP300, were positively correlated with the measured cell growth rate, whereas transcription factor motifs known to inhibit growth or induce apoptosis (e.g., PPARG, SREBPF, and STAT2,4,6) were negatively correlated (Fig. 3D; Supplemental Fig. S4A; Supplemental Table
S6). Moreover, correlations of growth with GSEA pathways (Fig. 3F; Supplemental Fig. S4B; Supplemental Table S6) or with FANTOM5 coexpression clusters (Supplemental Fig. S4C) showed that cell growth and replication-related pathways were positively correlated with the measured growth rate, whereas those related 
A

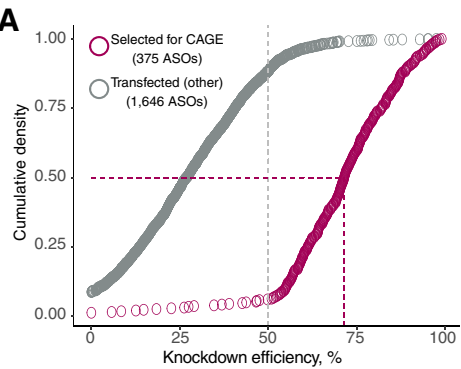

C

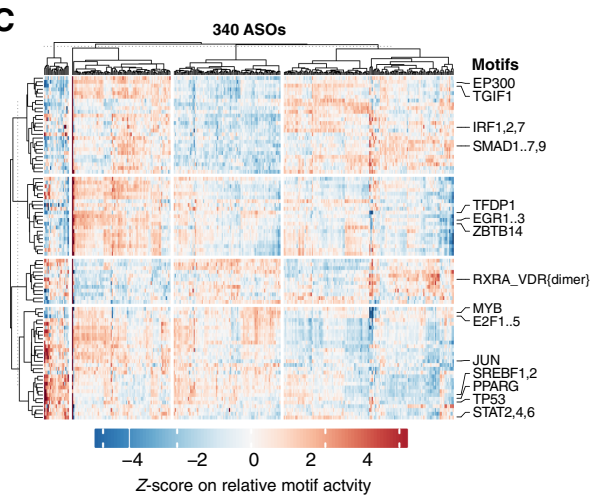

$\mathbf{E}$

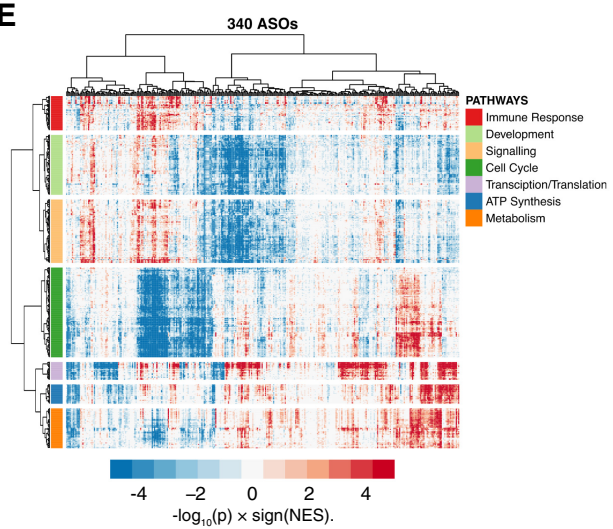

B

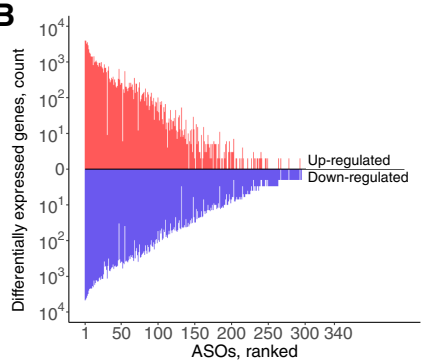

D

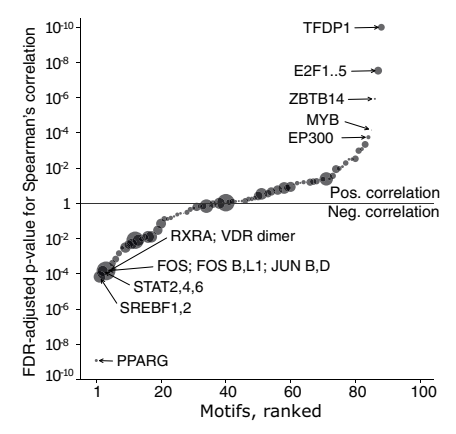

$\mathbf{F}$

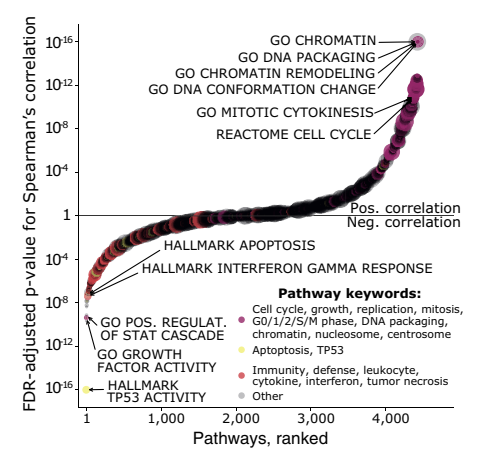

Figure 3. CAGE predicts cellular phenotypes. (A) RT-qPCR knockdown efficiency for 2021 ASO-transfected samples (targeted IncRNAs only). Gray dashed line indicates 50\% KD efficiency generally required for CAGE selection. Purple dashed lines indicate median KD efficiency (71.5\%) for 375 ASOs selected for CAGE sequencing. After quality control, 340 ASOs targeting IncRNAs were included for further analysis. (B) Distribution of significantly differentially expressed genes (up-regulated: $F D R<0.05, Z$-score $>1.645$, $\log _{2} \mathrm{FC}>0.5$; and down-regulated: $\mathrm{FDR}<0.05, Z$-score $\left.<-1.645, \log _{2} \mathrm{FC}<-0.5\right)$ across all 340 ASOs. (C) Motif Response Activity Analysis (MARA) across 340 ASOs. Scale indicates Z-score of the relative motif activity (the range was set to abs[Z-score] $=<5$ for visualization purposes). (D) Correlation between normalized growth rate and motif activities across 340 ASOs targeting IncRNAs with highlighted examples. Motif sizes shown are scaled based on the HDF expression of their associated TFs (range 1 to $\sim 600$ TPM). (E) Enriched biological pathways across 340 ASOs. Scale indicates GSEA enrichment value calculated as $-\log _{10}(p) \times \operatorname{sign}(N E S)$. ( $\left.F\right)$ Same as in $D$ but for selected GSEA pathways. Pathways sizes are scaled based on the number of associated genes.

to immunity, and cell stress and cell death were negatively correlated. We found that among 53 ASOs implicated in a growth-inhibition pathway based on the CAGE profiles, only $43 \%$ of them showed growth inhibition in the real-time imaging. This might suggest better sensitivity of transcriptomic profiling when detecting phenotypes as compared to live cell imaging methods, which are more prone to a delayed cellular response to the knockdown.

Additionally, morphological changes were reflected in the molecular phenotype assessed by CAGE (Supplemental Fig. S4D).
Cell radius and axis length were associated with GSEA categories related to actin arrangement and cilia, whereas cell compactness was negatively correlated with apoptosis. The extensive molecular phenotyping analysis also revealed pathways not explicitly associated with cell growth and cell morphology, such as transcription, translation, metabolism, development, and signaling (Fig. 3E).

Next, to globally assess whether individual ASO knockdowns lead to lncRNA-specific effects, we scaled the expression change of each gene across the whole experiment and compared differentially expressed genes (Fig. 3B) of all possible ASO pairs targeting the same lncRNA target versus different lncRNAs (Supplemental Methods; Supplemental Table S5). We found that the concordance of the same target group was significantly greater than that of the different target group (comparing the Jaccard indices across 10,000 permutations) (Supplemental Fig. S5A), suggesting that ASO knockdowns are nonrandom and lead to more lncRNA specific effects than the nontargeting ASO pairs. Further, by requiring at least five common DEGs (FDR $\leq 0.05$, abs $\left[\log _{2} \mathrm{FC}\right]>0.5$, abs $[Z$-score $\left.]>1.645\right)$ and ASO-pairs significantly above the nontargeting ASO pairs background ( $P \leq$ 0.05 ), we identified 16 ASO pairs, targeting 13 lncRNAs, exhibiting reproducible knockdown-mediated molecular responses in human dermal fibroblasts (Supplemental Fig. S5B). Corresponding GSEA pathways and MARA motifs of these 16 ASO pairs are shown in Supplemental Figure S5C.

\section{siRNA validation experiments}

To evaluate whether the lncRNA-specific effects can be measured by other knockdown technologies, nine lncRNAs, with relatively mild growth phenotype, were subjected to siRNA knockdown. Measuring transcriptional response, we noted that higher concordance was observed for ASO modality alone (Supplemental Fig. S5D). The observed discrepancies in the transcriptional response between ASO- and siRNA-mediated knockdowns could be contributed by their mode of action and variable activities in different subcellular compartments. Next, a concordant response was found for (5/36) ASO-siRNA pairs targeting three lncRNAs (Supplemental Fig. S5E; Supplemental Table S5), enriched in the cytoplasm (MAPKAPK5-AS1), soluble nuclear fraction (LINCO2454), and in the chromatin-bound fraction (A1BG-AS1). Although we cannot completely exclude the technical artifacts of each technology, concordant cellular response 
A

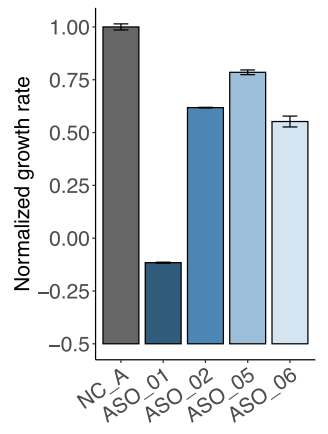

D

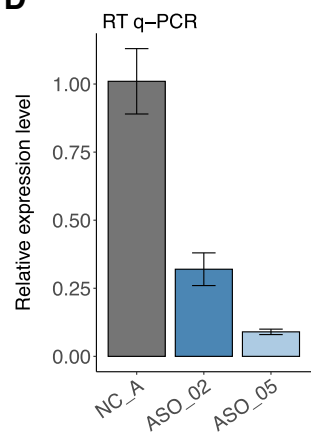

B

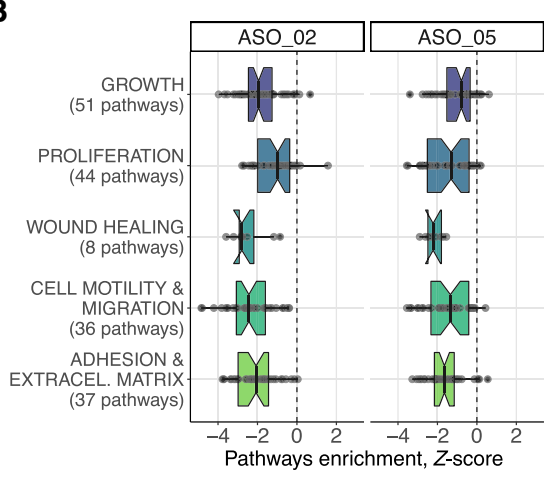

E

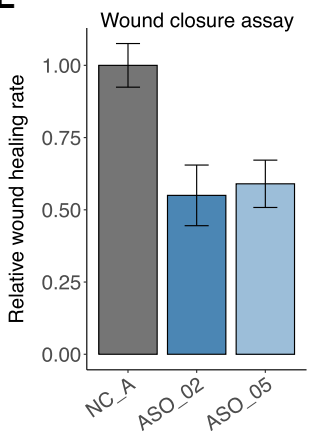

Negative Control
C
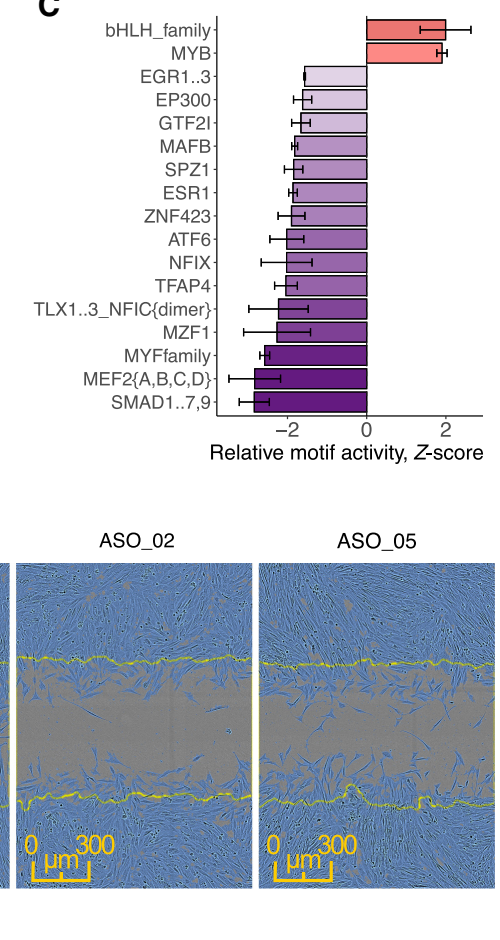

Figure 4. ZNF213-AS1 regulates cell growth, migration, and proliferation. (A) Normalized growth rate across four distinct ASOs (in duplicate) targeting ZNF213-AS1 as compared to six negative control samples (shown in gray). (B) Enrichment of biological pathways associated with growth, proliferation, wound healing, migration, and adhesion for ASO_02 and ASO_05. (C) Most consistently down- and up-regulated transcription factor binding motifs including those for transcription factors known to modulate growth, migration, and proliferation such as for example EGR family, EP300, GTF2I. $(D)$ Knockdown efficiency measured by RT-qPCR after wound closure assay (72 h posttransfection) showing sustained suppression (65\%-90\%) of ZNF213-AS1. (E) Transfected, replated, and mitomycin C (5 $\mu \mathrm{g} / \mathrm{mL})$-treated HDF cells were scratched and monitored in the Incucyte imaging system. Relative wound closure rate calculated during the $24 \mathrm{~h}$ postscratching shows $40 \%-45 \%$ reduction for the two targeting ASOs (ASO_02 [N=10] and ASO_05 [N=13]) as compared to NC_A transfection controls $(N=33$, shown in gray) and the representative images of wound closure assay $16 \mathrm{~h}$ postscratching.

exhibited by using ASOs alone suggests that lncRNAs, in part, are essential regulatory elements in cells. Yet, our study generally warrants a careful assessment of specific findings from different knockdown technologies, including CRISPR-inhibition, and demonstrates a requirement of using multiple replicates in a given target per each modality.

\section{ZNF213-AS1 is associated with cell growth and migration}

Extensive molecular and cellular phenotype data for each ASO knockdown can be explored using our portal https://fantom.gsc .riken.jp/zenbu/reports/\#FANTOM6. As an example of an lncRNA associated with cell growth and morphology (Fig. 2G), we showcase ZNF213-AS1 (RP11-473M20.14). This IncRNA is highly conserved in placental mammals, moderately expressed ( eight CAGE tags per million) in HDFs, and enriched in the chromatin-bound fraction. Four distinct ASOs (ASO_01, ASO_02, ASO_05, and ASO_06) strongly suppressed expression of ZNF213-AS1, whereas expression of the ZNF213 sense gene was not significantly affected in any of the knockdowns. The four ASOs caused varying degrees of cell growth inhibition (Fig. 4A). ASO_01 and ASO_06 showed a reduction in cell number, as well as an up-regulation of apoptosis and immune and defense pathways in GSEA, suggesting cell death. While cell growth inhibition observed for ASO_02 and ASO_05 was confirmed by MKI67 marker staining (Fig. 2D; Supplemental Table
S7), the molecular phenotype revealed suppression of GSEA pathways related to cell growth, as well as to cell proliferation, motility, and extracellular structure organization (Fig. 4B). We also observed consistent down-regulation of motifs related to the observed cellular phenotype, for example, EGR1, EP300, SMAD1...7,9 (Fig. 4C).

As cell motility pathways were affected by the knockdown, we tested whether ZNF213-AS1 could influence cell migration. Based on the wound-closure assay after transient cell growth inhibition (mitomycin C and serum starvation) (Supplemental Fig. S2F,G), we observed a substantial reduction of wound closure rate $(\sim 40 \%$ over a 24-h period) in the ZNF213-AS1-depleted HDFs (Fig. 4D, E). The reduced wound healing rate should thus mainly reflect reduced cell motility, further confirming affected motility pathways predicted by the molecular phenotype.

As these results indicated a potential role of ZNF213-AS1 in cell growth and migration, we used FANTOM CAT Recount 2 atlas (Imada et al. 2020), which incorporates The Cancer Genome Atlas (TCGA) data set (Collado-Torres et al. 2017), and found relatively higher expression of ZNF213-AS1 in acute myeloid leukemia (LAML) and in low-grade gliomas (LGG) as compared to other cancers (Supplemental Fig. S6A). In LAML, the highest expression levels were associated with mostly undifferentiated states, whereas in LGG, elevated expression levels were found in oligodendrogliomas, astrocytomas, and in IDH1 mutated tumors, suggesting that ZNF213-AS1 is involved in modulating

\section{Genome Research}

www.genome.org 
differentiation and proliferation of tumors (Supplemental Fig. S6B-E). Further, univariate Cox proportional hazard analysis as well as Kaplan-Meier curves for LGG were significant and consistent with our findings $(\mathrm{HR}=0.61, \mathrm{BH} F \mathrm{FD}=0.0079)$. The same survival analysis on LAML showed a weak association with poor prognostic outcome, but the results were not significant (Supplemental Fig. S6F,G).

\section{RP11-398K22.12 (KHDC3L-2) regulates KCNQ5 in cis}

Next, we investigated in detail RP11-398K22.12 (ENSG00000229 852), where the knockdowns by two independent ASOs (ASO_03, ASO_05) successfully reduced the expression of the target lncRNA (67\%-82\% knockdown efficiency, respectively) and further down-regulated its neighboring genes, KCNQ5 and its divergent partner novel lncRNA CATG00000088862.1 (Fig. 5A). Although the two genomic loci occupy Chromosome 6 and are $650 \mathrm{~kb}$ away, Hi-C analysis (Supplemental Methods; Supplemental Fig. S7; Supplemental Table S8) showed that they are located within the same topologically associated domain (TAD) and spatially colocalized (Fig. 5B). Moreover, chromatin-enrichment and single molecule RNA-FISH of RP11-398K22.12 (Fig. 5C; Supplemental Table S9) suggested its highly localized cis-regulatory role.

In FANTOM5 (Hon et al. 2017), expression levels of RP11398K22.12, KCNQ5, and CATG00000088862.1 were enriched in brain and nervous system samples, whereas GTEx (The GTEx Consortium 2015) showed their highly specific expression in the brain, particularly in the cerebellum and the cerebellar hemisphere (Fig. 5D). GTEx data also showed that expression of RP11398K22.12 was highly correlated with the expression of KCNQ5 and CATG00000088862.1 across neuronal tissues (Fig. 5E,F), with the exception of cerebellum and the cerebellar hemisphere, potentially due to relatively lower levels of KCNQ5 and CATG00000088862.1, whereas levels of RP11-398K22.12 remained relatively higher. Additionally, we found an eQTL SNP (rs14526472) overlapping with RP11-398K22.12 and regulating expression of $K C N Q 5$ in brain caudate $\left(P=4.2 \times 10^{-6}\right.$; normalized effect size -0.58). All these findings indicate that $R P 11-398 K 22.12$ is implicated in the nervous system by maintaining the expression of KCNQ5 and CATG000 00088862.1 in a cis-acting manner.

\section{Discussion}

This study systematically annotates lncRNAs through molecular and cellular phenotyping by selecting 285 lncRNAs from human dermal fibroblasts across a wide spectrum of expression, conservation levels and subcellular localization enrichments. Using ASO technology allowed observed phenotypes to be associated to the IncRNA transcripts, whereas, in contrast, CRISPR-based approaches may synchronically influence the transcription machinery at the site of the divergent promoter or affect regulatory elements of the targeted DNA site. Knockdown efficiencies obtained with ASOs were observed to be independent of IncRNA expression levels, subcellular localization, and of their genomic annotation, allowing us to apply the same knockdown technology to various classes of lncRNAs.

We investigated the cis-regulation of nearby divergent promoters, which has been reported as one of the functional roles of lncRNA (Luo et al. 2016). However, in agreement with previous studies (Guttman et al. 2011), we did not observe general patterns in the expression response of divergent promoters (Supplemental Fig. S3B). Recent studies suggest that transcription of IncRNA loci that do not overlap with other transcription units may influence RNA polymerase II occupancy on neighboring promoters and gene bodies (Engreitz et al. 2016a; Cho et al. 2018). Thus, it is plausible that transcription of targeted lncRNA was maintained, despite suppression of mature or nascent transcripts using ASOs. This further suggests that the functional responses described in this study are due to interference of processed transcripts present either in the nucleus, the cytoplasm, or both. Although it is arguable that ASOs may interfere with general transcription by targeting the $5^{\prime}$-end of nascent transcripts and thus releasing RNA polymerase II, followed by exonuclease-mediated decay and transcription termination (aka "torpedo model") (Proudfoot 2016), most of the ASOs were designed across the entire length of the transcript. Since we did not broadly observe dysregulation in nearby genes, interference of transcription or splicing activity is less likely to occur.

We observed a reduction in cell growth for $\sim 7.7 \%$ of our target lncRNA genes, which is in line with previous experiments using CRISPRi-pooled screening, which reported 5.9\% (in iPS cells) of lncRNAs exhibiting a cell growth phenotype (Liu et al. 2017). Although these rates are much lower than for protein-coding genes (Sokolova et al. 2017), recurrent observations of cell growth phenotypes (including cell death) strongly suggest that a substantial fraction of lncRNAs play an essential role in cellular physiology and viability. Further, when applying image-based analysis, we found that lncRNAs affect cell morphologies (Fig. 2G), which has not been so far thoroughly explored.

Several lncRNAs such as MALAT1, NEAT1, and FIRRE have been reported to orchestrate transcription, RNA processing, and gene expression (Kopp and Mendell 2018) but are not essential for mouse development or viability. These observations advocate for assays that can comprehensively profile the molecular changes inside perturbed cells. Therefore, in contrast to cell-based assays, functional elucidation via molecular phenotyping provides comprehensive information that cannot be captured by a single phenotypic assay. Herein, the number of overlapping differentially expressed genes between two ASOs of the same lncRNA targets indicated that $10.9 \%$ of IncRNAs exert a reproducible regulatory function in HDF.

Although the features of selected lncRNAs are generally similar to those of other IncRNAs expressed in HDFs (Fig. 1B-D), the cell-type-specific nature of IncRNAs and the relatively small sampling size (119 lncRNAs with knockdown transcriptome profiles) used in our study may not fully represent the whole extent of lncRNA in other cell types. However, lncRNA targets that did not exhibit a molecular phenotype may be biologically relevant in other cell types or cell states (Li and Chang 2014; Liu et al. 2017). At the same time, our results showed that particular lncRNAs expressed broadly in other tissues (e.g., in the human brain) were functional in HDFs (such as RP11-398K22.12). Although the exact molecular mechanisms of $R P 11-398 K 22.12$ are not yet fully understood, its potential role in HDFs suggests that lncRNAs may be functionally relevant across multiple tissues in spite of the celltype-specific expression of IncRNAs.

Further, we used siRNA technology to knockdown lncRNA targets as a method for independent validation. When comparing the transcriptomes perturbed by ASOs and siRNAs, concordance was observed only for three out of nine lncRNAs. This discrepancy is likely due to different modes of actions of the two technologies. Whereas ASOs invoke RNase H-mediated cleavage, primarily active 
A
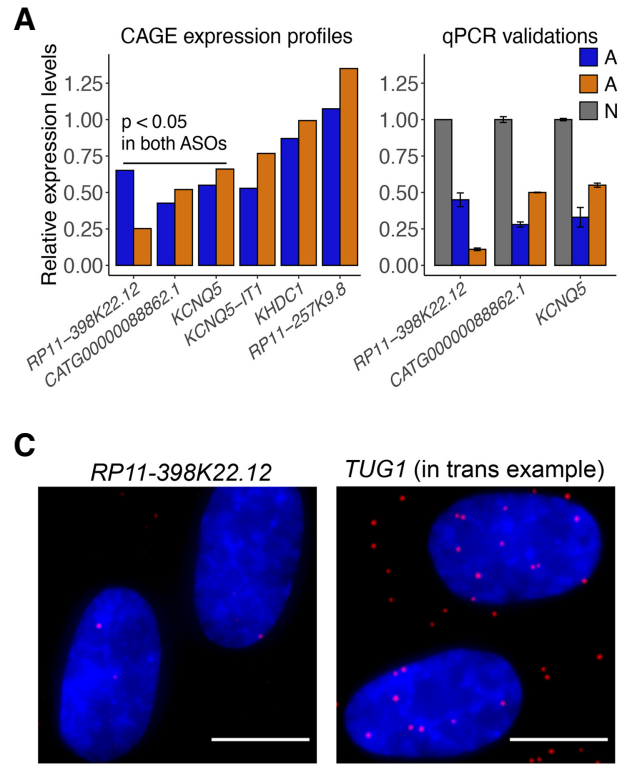

D

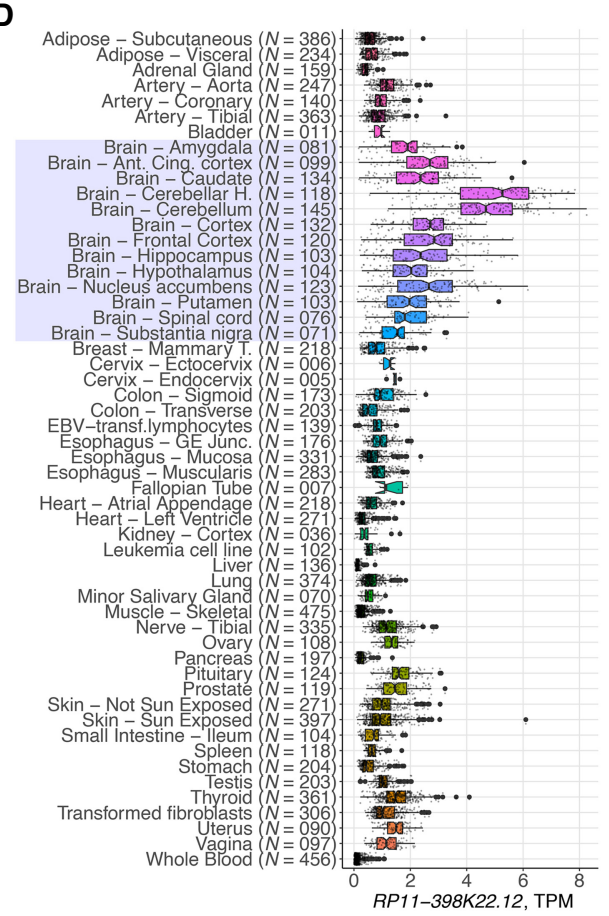

B

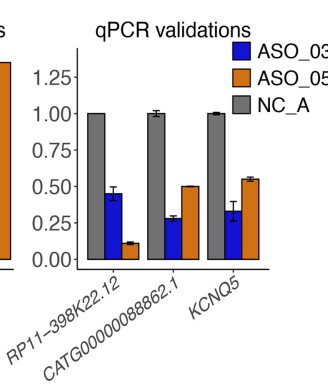

Hi-C heatmap

(25kb resolution)

0
20
Contact frequency
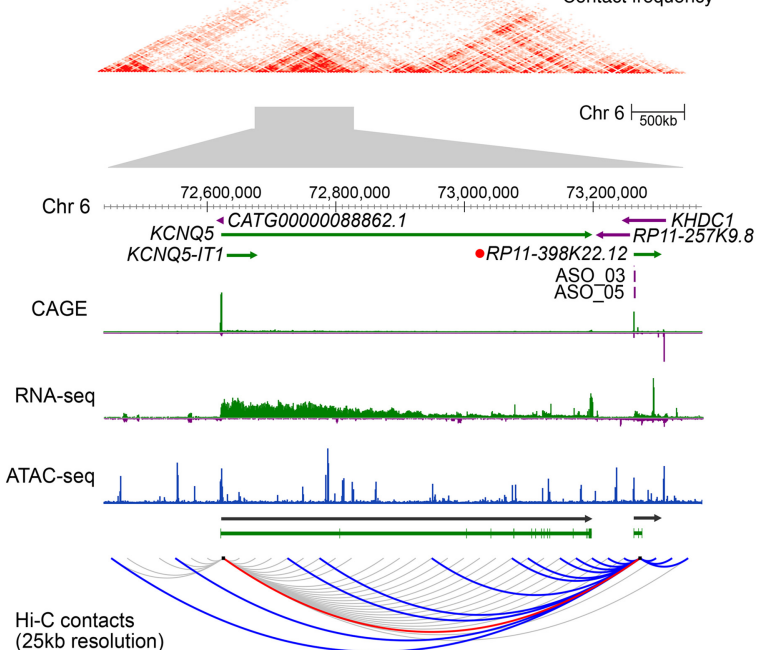
(25kb resolution)

E

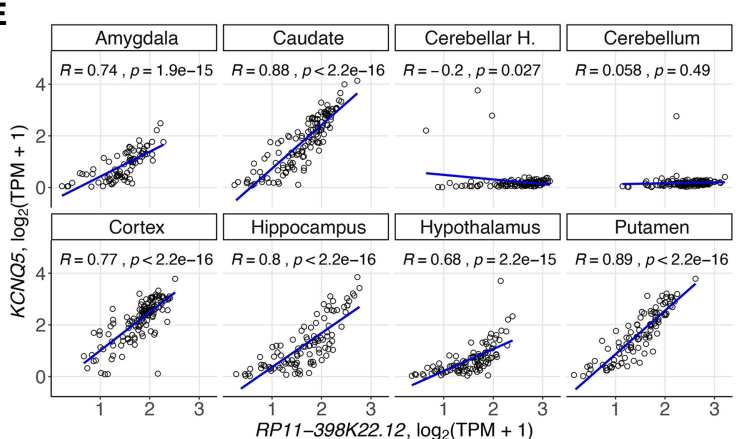

$\mathbf{F}$

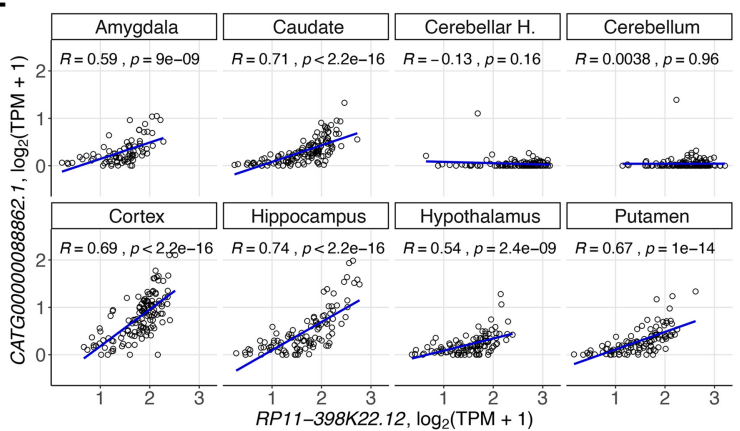

Figure 5. RP11-398K22.12 down-regulates KCNQ5 and CATG00000088862.1 in cis. (A) Changes in expression levels of detectable genes in the same topologically associated domain (TAD) as RP11-398K22.12 based on Hi-C analysis. Both KCNQ5 and CATG00000088862.1 are down-regulated $(P<0.05)$ upon the knockdown of RP11-398K22.12 by two independent ASOs in CAGE analysis (left) as further confirmed with RT-qPCR (right). (B) (Top) Representation of the chromatin conformation in the 4-Mb region proximal to the TAD containing RP11-398K22.12, followed by the locus gene annotation, CAGE, RNA-seq, and ATAC-seq data for native HDFs. (Bottom) Schematic diagram showing Hi-C predicted contacts of RP11-398K22.12 (blue) and KCNQ5 (gray) (25-kb resolution, frequency $\geq 5$ ) in HDF cells. Red line indicates RP11-398K22.12 and KCNQ5 contact. (C) FISH image for RP11-398K22.12, suggesting proximal regulation. TUG1 FISH image (suggesting trans regulation) is included as a comparison; $(\mathrm{bar}=10 \mu \mathrm{m})$. $(D)$ GTEx atlas across 54 tissues $(N=9662$ samples) shows relatively high expression levels of $R P 11-398 K 22.12$ in 13 distinct brain regions samples (highlighted). (E) Expression correlation for RP11-398K22.12 and KCNQ5 in eight out of 13 distinct brain regions, as highlighted in D. (F) Expression correlation for RP11-398K22.12 and CATC00000088862.1 in eight out of 13 distinct brain regions, as highlighted in $D$.

in the nucleus, the siRNAs use the RNA-inducing silencing complex (RISC) mainly active in the cytoplasm. LncRNAs are known to function in specific subcellular compartments (Chen 2016) and their maturity, secondary structures, isoforms, and functions could be vastly different across compartments (Johnsson et al. 2013). Since the majority of functional lncRNAs are reported to be inside the nucleus (Palazzo and Lee 2018; Sun et al. 2018), ASO-mediated knockdowns, which mainly target nuclear RNAs,

\section{Genome Research}

www.genome.org 
are generally more suitable for functional screenings of our IncRNA ( $62 \%$ found in the nuclear compartment). Besides, the dynamics of secondary effects mediated by different levels of knockdown from different technologies are likely to be observed as discordance when considering the whole transcriptome, where this kind of discordance has been reported previously (Stojic et al. 2018). In contrast, in the MKI67 assay, where only a single feature such as growth phenotype is assayed, siRNA knockdown revealed higher reproducibility with ASO knockdown. This suggested that the growth phenotype might be triggered by different specific pathways in ASO- and siRNA-knockdowns.

Previous studies suggest that lncRNAs regulate gene expression in trans epigenetically, via direct or indirect interaction with regulators such as DNMT1 (Di Ruscio et al. 2013) or by directly binding to DNA (triplex) (Mondal et al. 2015) or other RNA-binding proteins (Tichon et al. 2016). Analysis of cellular localization by fractionation followed by RNA-seq and in situ hybridization can indicate whether a given lncRNA may act in trans by quantifying its abundance in the nuclear soluble fraction as compared to cytoplasm. Although most lncRNAs in the nuclear soluble fraction may affect pathways associated with chromatin modification, additional experiments to globally understand their interaction partners will elucidate the molecular mechanism behind trans-acting IncRNAs (Li et al. 2017; Sridhar et al. 2017).

In summary, our study highlights the functional importance of IncRNAs regardless of their expression, localization, and conservation levels. Molecular phenotyping is a powerful and generally more sensitive to knockdown-mediated changes platform to reveal the functional relevance of lncRNAs that cannot be observed based on the cellular phenotypes alone. With additional molecular profiling techniques, such as RNA duplex maps in living cells to decode common structural motifs (Lu et al. 2016), and Oxford Nanopore Technology (ONT) to annotate the full-length variant isoforms of lncRNAs (Hardwick et al. 2019), the structure-to-functional relationship of lncRNAs may be elucidated further in the future.

\section{Methods}

\section{Gene models and IncRNA target selections}

The gene models used in this study were primarily based on the FANTOM CAGE-associated transcriptome (CAT) at permissive level as defined previously (Hon et al. 2017). From this merged assembly, there were 2000 lncRNAs robustly expressed in HDFs (TPM $\geq 1)$. However, we selected lncRNA knockdown targets in an unbiased manner to broadly cover various types of lncRNAs (TPM $\geq 0.2$ ). Briefly, we first identified a list of the lncRNA genes expressed in HDFs, with RNA-seq expression at least 0.5 fragments per kilobase per million and CAGE expression at least 1 tag per million. Then, we manually inspected each lncRNA locus in the ZENBU genome browser for (1) its independence from neighboring genes on the same strand (if any), (2) support from RNA-seq (for exons and splicing junctions) and CAGE data (for TSSs) of its transcript models, and (3) support from histone marks at TSSs for transcription initiation (H3K27ac) and along the gene body for elongation (H3K36me3), from the Roadmap Epigenomics Consortium (Roadmap Epigenomics Consortium et al. 2015). A representative transcript model, which best represents the RNAseq signal, was manually chosen from each locus for design of antisense oligonucleotides. In total, 285 lncRNA loci were chosen for ASO suppression. Additional controls (NEAT1, protein coding genes) (Supplemental Table S1) were added, including MALAT1 as an experimental control. For details, please refer to the Supplemental Methods.

\section{ASO design}

ASOs were designed as RNase H-recruiting locked nucleic acid (LNA) phosphorothioate gapmers with a central DNA gap flanked by $2-4$ LNA nucleotides at the $5^{\prime}$ and $3^{\prime}$ ends of the ASOs. For details, please refer to the Supplemental Methods.

\section{Automated cell culturing, ASO transfection, and cell harvesting}

Robotic automation (Hamilton) was established to provide a stable environment and accurate procedural timing control for cell culturing and transfection. In brief, trypsin-EDTA detachment, cell number and viability quantification, cell seeding, transfection, and cell harvesting were performed with automation. All transfections were divided into 28 runs on a weekly basis. ASO transfection was performed with duplication. In each run, there were 16 independent transfections with ASO negative control A (NC_A, Exiqon) and 16 wells transfected with an ASO targeting MALAT1 (Exiqon).

The HDF cells were seeded in 12-well plates with 80,000 cells in each well $24 \mathrm{~h}$ prior to the transfection. A final concentration of $20 \mathrm{nM}$ ASO and $2 \mu \mathrm{L}$ Lipofectamine RNAiMAX (Thermo Fisher Scientific) were mixed in $200 \mu \mathrm{L}$ Opti-MEM (Thermo Fisher Scientific). The mixture was incubated at room temperature for $5 \mathrm{~min}$ and added to the cells, which were maintained in $1 \mathrm{~mL}$ complete medium. The cells were harvested $48 \mathrm{~h}$ posttransfection by adding $200 \mu \mathrm{L}$ RLT buffer from the RNeasy 96 kit (Qiagen) after PBS washing. The harvested lysates were kept at $-80^{\circ} \mathrm{C}$. RNA was extracted from the lysate for real-time quantitative RT-PCR (Supplemental Methods).

\section{ASO transfection for real-time imaging}

The HDF cells were transfected manually in 96-well plates to facilitate high-throughput real-time imaging. The cells were seeded $24 \mathrm{~h}$ before transfection at a density of 5200 cells per well. A final concentration of $20 \mathrm{nM}$ ASO and $2 \mu \mathrm{L}$ Lipofectamine RNAiMAX (Thermo Fisher Scientific) were mixed in $200 \mu \mathrm{L}$ Opti-MEM (Thermo Fisher Scientific). After incubating at room temperature for $5 \mathrm{~min}, 18 \mu \mathrm{L}$ of the transfection mix was added to $90 \mu \mathrm{L}$ complete medium in each well. The ASOs were divided into 14 runs and transfected in duplicate. Each plate accommodated six wells of NC_A control, two wells of MALAT1 ASO control, and two wells of mock-transfection (Lipofectamine alone) control.

Phase-contrast images of transfected cells were captured every $3 \mathrm{~h}$ for $2 \mathrm{~d}$ with three fields per well by the Incucyte live-cell imaging system (Essen Bioscience). The confluence in each field was analyzed by the Incucyte software. The mean confluence of each well was taken along the timeline until the mean confluence of the NC_A control in the same plate reached $90 \%$. The growth rate in each well was calculated as the slope of a linear regression. A normalized growth rate of each replicate was calculated as the growth rate divided by the mean growth rate of the six NC_A controls from the same plate. Negative growth rate was derived when cells shrink and/or detach. As these rates of cell depletion could not be normalized by the rate of growth, negative values were maintained to indicate severe growth inhibition. Student's $t$-test was performed between the growth rate of the duplicated samples and the six NC_A controls, assuming equal variance. 


\section{Cell morphology quantification}

For each transfection, a representative phase-contrast image at a single time point was exported from the Incucyte time-series. These raw images were first transformed to probability maps of cells by pixel classification using ilastik (1.3.2) (Berg et al. 2019). The trained model was then applied to all images where the predicted probability maps of cells (grayscale, 16 bits tiff format) were subsequently used for morphology quantification in CellProfiler (3.1.5) (Carpenter et al. 2006). For details, please refer to the Supplemental Methods.

\section{MKI67 staining upon IncRNA knockdown}

For the selected four lncRNA targets showing $>25 \%$ growth inhibition, we used two siRNAs and two ASOs with independent sequences. The transfected cells were fixed by adding prechilled $70 \%$ ethanol and incubated at $-20^{\circ} \mathrm{C}$. The cells were washed with FACS buffer (2\% FBS in PBS, 0.05\% NaN3) twice. FITC-conjugated MKI67 (20Raj1, eBioscience) was applied to the cells and subjected to flow cytometric analysis. Knockdown efficiency by siRNA was determined by real-time quantitative RT-PCR using the same three primer pairs as for ASO knockdown efficiency. For details, please refer to the Supplemental Methods.

\section{Wound closure assay}

The HDF cells were transfected with $20 \mathrm{nM}$ ASO as described earlier in 12-well plates. The cells were replated at $24 \mathrm{~h}$ posttransfection into a 96-well ImageLock plate (Essen BioScience) at a density of 20,000 cells per well. At $24 \mathrm{~h}$ after seeding, cells form a spatially uniform monolayer with $95 \%-100 \%$ cell confluence. The cells were incubated with $5 \mu \mathrm{g} / \mathrm{mL}$ mitomycin $\mathrm{C}$ for $2 \mathrm{~h}$ to inhibit cell division. Then, medium was refreshed and a uniform scratch was created in each well by the WoundMaker (Essen BioScience). The closure of the wound was monitored by Incucyte live-cell imaging system (Essen Bioscience) every $2 \mathrm{~h}$ for $24 \mathrm{~h}$. The RNA was harvested after the assay for real-time quantitative RT-PCR. For details, please refer to the Supplemental Methods.

\section{Cap analysis of gene expression (CAGE)}

Four micrograms of purified RNA were used to generate libraries according to the nAnT-iCAGE protocol (Murata et al. 2014). For details, please refer to the Supplemental Methods.

\section{Chromosome conformation capture (Hi-C)}

$\mathrm{Hi}-\mathrm{C}$ libraries were prepared essentially as described previously (Lieberman-Aiden et al. 2009; Fraser et al. 2015a) with minor changes to improve the DNA yield of Hi-C products (Fraser et al. 2015b). For details, please refer to the Supplemental Methods.

\section{Data access}

All raw and processed sequencing data generated in this study have been submitted to the DNA Data Bank of Japan (DDBJ; https:// www.ddbj.nig.ac.jp/) under accession numbers DRA008311, DRA008312, DRA008436, and DRA008511 or can be accessed through the FANTOM6 project portal https://fantom.gsc.riken .jp/6/datafiles. The analysis results can be downloaded from https://fantom.gsc.riken.jp/6/suppl/Ramilowski_et_al_2020/data/ and interactively explored using our in-house portal https ://fantom.gsc.riken.jp/zenbu/reports/\#FANTOM6.

\section{Competing interest statement}

The authors declare no competing interests.

\section{Acknowledgments}

We thank Linda Kostrencic, Hiroto Atsui, Emi Ito, Nobuyuki Takeda, Tsutomu Saito, Teruaki Kitakura, Yumi Hara, Machiko Kashiwagi, and Masaaki Furuno at RIKEN Yokohama for assistance in arranging collaboration agreements, ethics applications, computational infrastructure, and the FANTOM6 meetings. We also thank RIKEN GeNAS for generation and sequencing of the CAGE libraries and subsequent data processing. FANTOM6 was made possible by a Research Grant for RIKEN Center for Life Science Technology, Division of Genomic Technologies (CLST DGT) and RIKEN Center for Integrative Medical Sciences (IMS) from MEXT, Japan. I.V.K. and I.E.V. were supported by Russian Foundation for Basic Research (RFBR) 18-34-20024, B.B. is supported by the fellowship 2017FI_B00722 from the Secretaria d'Universitats i Recerca del Departament d'Empresa i Coneixement (Generalitat de Catalunya) and the European Social Fund (ESF), A. Favorov was supported by National Institutes of Health (NIH) P30 CA006973 and RFBR 17-00-00208, D.G. is supported by a "la Caixa"-Severo Ochoa pre-doctoral fellowship (LCF/BQ/SO15/ 52260001), E.L.I. and L.M. were supported by NIH National Cancer Institute Grant R01CA200859 and Department of Defense (DOD) award W81XWH-16-1-0739, M.K.-S. was supported by Versus Arthritis UK 20298, A.L. was supported by the Swedish Cancer Society, The Swedish Research Council, the Swedish Childhood Cancer fund, Radiumhemmets forsknigsfonder; V.J.M. was supported by the Russian Academy of Sciences Project 0112-2019-0001; Y.A.M. was supported by Russian Science Foundation (RSF) grant 18-14-00240, A.S. was supported by Novo Nordisk Foundation, Lundbeck Foundation, Danish Cancer Society, Carlsberg Foundation, Independent Research Fund Denmark, A.R.R.F. is currently supported by an Australian National Health and Medical Research Council Fellowship APP1154524, M.M.H. was supported by Natural Sciences and Engineering Research Council of Canada (RGPIN2015-3948), C.S. was supported by the Interuniversity Consortium for Biotechnology (CIB) from the Italian Ministry of Education, University and Research (MIUR) grant n.974, CMPT177780. J. Luginbühl was supported by Japan Society for the Promotion of Science (JSPS) Postdoctoral Fellowship for Foreign Researchers. C.J.C.P. was supported by RIKEN Special Post-Doctoral Research (SPDR) fellowship.

\section{References}

Ambasudhan R, Talantova M, Coleman R, Yuan X, Zhu S, Lipton SA, Ding S 2011. Direct reprogramming of adult human fibroblasts to functional neurons under defined conditions. Cell Stem Cell 9: 113-118. doi:10 .1016/j.stem.2011.07.002

Bai J, Yao B, Wang L, Sun L, Chen T, Liu R, Yin G, Xu Q, Yang W. 2019. lncRNA A1BG-AS1 suppresses proliferation and invasion of hepatocellular carcinoma cells by targeting miR-216a-5p. J Cell Biochem 120: 10310-10322. doi:10.1002/jcb.28315

Berg S, Kutra D, Kroeger T, Straehle CN, Kausler BX, Haubold C, Schiegg M, Ales J, Beier T, Rudy M, et al. 2019. ilastik: interactive machine learning for (bio)image analysis. Nat Methods 16: 1226-1232. doi:10.1038/ s41592-019-0582-9

Carninci P, Kasukawa T, Katayama S, Gough J, Frith MC, Maeda N, Oyama R, Ravasi T, Lenhard B, Wells C, et al. 2005. The transcriptional landscape of the mammalian genome. Science 309: 1559-1563. doi:10 $.1126 /$ science. 1112014

Carpenter AE, Jones TR, Lamprecht MR, Clarke C, Kang I, Friman O, Guertin DA, Chang J, Lindquist RA, Moffat J, et al. 2006. CellProfiler: image 
analysis software for identifying and quantifying cell phenotypes. Genome Biol 7: R100. doi:10.1186/gb-2006-7-10-r100

Carrieri C, Cimatti L, Biagioli M, Beugnet A, Zucchelli S, Fedele S, Pesce E, Ferrer I, Collavin L, Santoro C, et al. 2012. Long non-coding antisense RNA controls Uchl1 translation through an embedded SINEB2 repeat. Nature 491: 454-457. doi:10.1038/nature11508

Chen L-L. 2016. Linking long noncoding RNA localization and function. Trends Biochem Sci 41: 761-772. doi:10.1016/j.tibs.2016.07.003

Cho SW, Xu J, Sun R, Mumbach MR, Carter AC, Chen YG, Yost KE, Kim J, He J, Nevins SA, et al. 2018. Promoter of lncRNA gene PVT1 is a tumor-suppressor DNA boundary element. Cell 173: 1398-1412.e22. doi:10.1016/ j.cell.2018.03.068

Chu C, Zhang QC, da Rocha ST, Flynn RA, Bharadwaj M, Calabrese JM, Magnuson T, Heard E, Chang HY. 2015. Systematic discovery of Xist RNA binding proteins. Cell 161: 404-416. doi:10.1016/j.cell.2015 .03 .025

Collado-Torres L, Nellore A, Kammers K, Ellis SE, Taub MA, Hansen KD, Jaffe AE, Langmead B, Leek JT. 2017. Reproducible RNA-seq analysis using recount2. Nat Biotechnol 35: 319-321. doi:10.1038/nbt.3838

De Hoon M, Shin JW, Carninci P. 2015. Paradigm shifts in genomics through the FANTOM projects. Mamm Genome 26: 391-402. doi:10 $.1007 / \mathrm{s} 00335-015-9593-8$

Di Ruscio A, Ebralidze AK, Benoukraf T, Amabile G, Goff LA, Terragni J, Figueroa ME, De Figueiredo Pontes LL, Alberich-Jorda M, Zhang P, et al. 2013. DNMT1-interacting RNAs block gene-specific DNA methylation. Nature 503: 371-376. doi:10.1038/nature12598

Engreitz JM, Haines JE, Perez EM, Munson G, Chen J, Kane M, McDonel PE, Guttman M, Lander ES. 2016a. Local regulation of gene expression by lncRNA promoters, transcription and splicing. Nature 539: 452-455. doi:10.1038/nature20149

Engreitz JM, Ollikainen N, Guttman M. 2016b. Long non-coding RNAs: spatial amplifiers that control nuclear structure and gene expression. Nat Rev Mol Cell Biol 17: 756-770. doi:10.1038/nrm.2016.126

The FANTOM Consortium, Suzuki H, Forrest ARR, Van Nimwegen E, Daub CO, Balwierz PJ, Irvine KM, Lassmann T, Ravasi T, Hasegawa Y, et al. 2009. The transcriptional network that controls growth arrest and differentiation in a human myeloid leukemia cell line. Nat Genet 41: 553-562. doi:10.1038/ng.375

Fraser J, Ferrai C, Chiariello AM, Schueler M, Rito T, Laudanno G, Barbieri M, Moore BL, Kraemer DCA, Aitken S, et al. 2015a. Hierarchical folding and reorganization of chromosomes are linked to transcriptional changes in cellular differentiation. Mol Syst Biol 11: 852 . doi:10.15252/msb .20156492

Fraser J, Williamson I, Bickmore WA, Dostie J. 2015b. An overview of genome organization and how we got there: from FISH to Hi-C. Microbiol Mol Biol Rev 79: 347-372. doi:10.1128/MMBR.00006-15

The GTEx Consortium. 2015. Human genomics. The Genotype-Tissue Expression (GTEx) pilot analysis: multitissue gene regulation in humans. Science 348: 648-660. doi:10.1126/science.1262110

Gupta RA, Shah N, Wang KC, Kim J, Horlings HM, Wong DJ, Tsai M-C, Hung T, Argani P, Rinn JL, et al. 2010. Long non-coding RNA HOTAIR reprograms chromatin state to promote cancer metastasis. Nature 464: 1071-1076. doi:10.1038/nature08975

Guttman M, Rinn JL. 2012. Modular regulatory principles of large non-coding RNAs. Nature 482: 339-346. doi:10.1038/nature10887

Guttman M, Donaghey J, Carey BW, Garber M, Grenier JK., Munson G, Young G, Lucas AB, Ach R, Bruhn L, et al. 2011. lincRNAs act in the circuitry controlling pluripotency and differentiation. Nature 477: 295300. doi:10.1038/nature10398

Hardwick SA, Bassett SD, Kaczorowski D, Blackburn J, Barton K, Bartonicek N, Carswell SL, Tilgner HU, Loy C, Halliday G, et al. 2019. Targeted, high-resolution RNA sequencing of non-coding genomic regions associated with neuropsychiatric functions. Front Genet 10: 309. doi:10.3389/ fgene.2019.00309

Hon C-C, Ramilowski JA, Harshbarger J, Bertin N, Rackham OJL, Gough J, Denisenko E, Schmeier S, Poulsen TM, Severin J, et al. 2017. An atlas of human long non-coding RNAs with accurate $5^{\prime}$ ends. Nature 543: 199-204. doi:10.1038/nature21374

Imada E-L, Sanchez DF, Collado-Torres L, Wilks C, Matam T, Dinalankara W, Stupnikov A, Lobo-Pereira F, Yip C-W, Yasuzawa K, et al. 2020. Recounting the FANTOM CAGE-Associated Transcriptome. Genome Res (this issue). doi:10.1101/gr.254656.119

Iyer MK, Niknafs YS, Malik R, Singhal U, Sahu A, Hosono Y, Barrette TR, Prensner JR, Evans JR, Zhao S, et al. 2015. The landscape of long noncoding RNAs in the human transcriptome. Nat Genet 47: 199-208. doi:10 $.1038 /$ ng.3192

Johnsson P, Ackley A, Vidarsdottir L, Lui W-O, Corcoran M, Grandér D, Morris KV. 2013. A pseudogene long-noncoding-RNA network regulates PTEN transcription and translation in human cells. Nat Struct Mol Biol 20: $440-446$. doi:10.1038/nsmb.2516
Joung J, Engreitz JM, Konermann S, Abudayyeh OO, Verdine VK, Aguet F, Gootenberg JS, Sanjana NE, Wright JB, Fulco CP, et al. 2017. Genomescale activation screen identifies a lncRNA locus regulating a gene neighbourhood. Nature 548: 343-346. doi:10.1038/nature23451

Kalluri R. 2016. The biology and function of fibroblasts in cancer. Nat Rev Cancer 16: 582-598. doi:10.1038/nrc.2016.73

Kendall RT, Feghali-Bostwick CA. 2014. Fibroblasts in fibrosis: novel roles and mediators. Front Pharmacol 5: 123. doi:10.3389/fphar.2014.00123

Kopp F, Mendell JT. 2018. Functional classification and experimental dissection of long noncoding RNAs. Cell 172: 393-407. doi:10.1016/ .cell.2018.01.011

Li L, Chang HY. 2014. Physiological roles of long noncoding RNAs: insight from knockout mice. Trends Cell Biol 24: 594-602. doi:10.1016/j.tcb 2014.06.003

Li B, Wang JH-C. 2011. Fibroblasts and myofibroblasts in wound healing: force generation and measurement. J Tissue Viability 20: 108-120. doi:10.1016/j.jtv.2009.11.004

Li X, Zhou B, Chen L, Gou L-T, Li H, Fu X-D. 2017. GRID-seq reveals the global RNA-chromatin interactome. Nat Biotechnol 35: 940-950. doi:10.1038/nbt.3968

Lieberman-Aiden E, van Berkum NL, Imakaev M, Ragoczy T, Telling A, Amit I, Lajoie BR, Sabo PJ, Dorschner MO, Williams L, et al. 2009. Comprehensive mapping of long-range interactions reveals folding principles of the human genome. Science 326: 289-293. doi:10.1126/sci ence.1181369

Liu SJ, Horlbeck MA, Cho SW, Birk HS, Malatesta M, He D, Attenello FJ, Villalta JE, Cho MY, Chen Y, et al. 2017. CRISPRi-based genome-scale identification of functional long noncoding RNA loci in human cells. Science 355: eaah7111. doi:10.1126/science.aah7111

Liu Y, Cao Z, Wang Y, Guo Y, Xu P, Yuan P, Liu Z, He Y, Wei W. 2018. Genome-wide screening for functional long noncoding RNAs in human cells by Cas9 targeting of splice sites. Nat Biotechnol 36: 1203-1210. doi: $10.1038 /$ nbt. 4283

Lu Z, Zhang QC, Lee B, Flynn RA, Smith MA, Robinson JT, Davidovich C, Gooding AR, Goodrich KJ, Mattick JS, et al. 2016. RNA duplex map in living cells reveals higher-order transcriptome structure. Cell 165: 1267-1279. doi:10.1016/j.cell.2016.04.028

Luo S, Lu JY, Liu L, Yin Y, Chen C, Han X, Wu B, Xu R, Liu W, Yan P, et al. 2016. Divergent lncRNAs regulate gene expression and lineage differentiation in pluripotent cells. Cell Stem Cell 18: 637-652. doi:10.1016/j stem.2016.01.024

Ma L, Cao J, Liu L, Du Q, Li Z, Zou D, Bajic VB, Zhang Z. 2019. LncBook: a curated knowledgebase of human long non-coding RNAs. Nucleic Acids Res 47: D128-D134. doi:10.1093/nar/gky960

Mondal T, Subhash S, Vaid R, Enroth S, Uday S, Reinius B, Mitra S, Mohammed A, James AR, Hoberg E, et al. 2015. MEG3 long noncoding RNA regulates the TGF- $\beta$ pathway genes through formation of RNADNA triplex structures. Nat Commun 6: 7743. doi:10.1038/ ncomms8743

Murata M, Nishiyori-Sueki H, Kojima-Ishiyama M, Carninci P, Hayashizaki Y, Itoh M. 2014. Detecting expressed genes using CAGE. Methods Mol Biol 1164: 67-85. doi:10.1007/978-1-4939-0805-9_7

Palazzo AF, Lee ES. 2018. Sequence determinants for nuclear retention and cytoplasmic export of mRNAs and lncRNAs. Front Genet 9: 440. doi:10 .3389 /fgene.2018.00440

Proudfoot NJ. 2016. Transcriptional termination in mammals: stopping the RNA polymerase II juggernaut. Science 352: aad9926. doi:10.1126/sci ence.aad9926

Quek XC, Thomson DW, Maag JLV, Bartonicek N, Signal B, Clark MB, Gloss BS, Dinger ME. 2015. IncRNAdb v2.0: expanding the reference database for functional long noncoding RNAs. Nucleic Acids Res 43: D168-D173. doi:10.1093/nar/gku988

Quinn JJ, Chang HY. 2016. Unique features of long non-coding RNA biogenesis and function. Nat Rev Genet 17: 47-62. doi:10.1038/nrg.2015.10

Ransohoff JD, Wei Y, Khavari PA. 2018. The functions and unique features of long intergenic non-coding RNA. Nat Rev Mol Cell Biol 19: 143-157. doi:10.1038/nrm.2017.104

Roadmap Epigenomics Consortium, Kundaje A, Meuleman W, Ernst J, Bilenky M, Yen A, Heravi-Moussavi A, Kheradpour P, Zhang Z, Wang $\mathrm{J}$, et al. 2015. Integrative analysis of 111 reference human epigenomes. Nature 518: $317-330$. doi: $10.1038 /$ nature 14248

Roux BT, Lindsay MA, Heward JA. 2017. Knockdown of nuclear-located enhancer RNAs and long ncRNAs using locked nucleic acid GapmeRs. Methods Mol Biol 1468: 11-18. doi:10.1007/978-1-4939-4035-6_2

Sokolova M, Turunen M, Mortusewicz O, Kivioja T, Herr P, Vähärautio A, Björklund M, Taipale M, Helleday T, Taipale J. 2017. Genome-wide screen of cell-cycle regulators in normal and tumor cells identifies a differential response to nucleosome depletion. Cell Cycle 16: 189-199. doi:10.1080/15384101.2016.1261765 
Sridhar B, Rivas-Astroza M, Nguyen TC, Chen W, Yan Z, Cao X, Hebert L, Zhong S. 2017. Systematic mapping of RNA-chromatin interactions in vivo. Curr Biol 27: 602-609. doi:10.1016/j.cub.2017.01.011

Stojic L, Lun ATL, Mangei J, Mascalchi P, Quarantotti V, Barr AR, Bakal C, Marioni JC, Gergely F, Odom DT. 2018. Specificity of RNAi, LNA and CRISPRi as loss-of-function methods in transcriptional analysis. Nucleic Acids Res 46: 5950-5966. doi:10.1093/nar/gky437

Subramanian A, Tamayo P, Mootha VK, Mukherjee S, Ebert BL, Gillette MA, Paulovich A, Pomeroy SL, Golub TR, Lander ES, et al. 2005. Gene set enrichment analysis: a knowledge-based approach for interpreting genome-wide expression profiles. Proc Natl Acad Sci 102: 15545-15550. doi:10.1073/pnas.0506580102

Sun Q, Hao Q, Prasanth KV. 2018. Nuclear long noncoding RNAs: key regulators of gene expression. Trends Genet 34: 142-157. doi:10.1016/j.tig 2017.11.005

Takahashi K, Tanabe K, Ohnuki M, Narita M, Ichisaka T, Tomoda K, Yamanaka S. 2007. Induction of pluripotent stem cells from adult human fibroblasts by defined factors. Cell 131: 861-872. doi:10.1016/j cell.2007.11.019
Tichon A, Gil N, Lubelsky Y, Havkin Solomon T, Lemze D, Itzkovitz S, SternGinossar N, Ulitsky I. 2016. A conserved abundant cytoplasmic long noncoding RNA modulates repression by Pumilio proteins in human cells. Nat Commun 7: 12209. doi:10.1038/ncomms12209

Ulitsky I. 2016. Evolution to the rescue: using comparative genomics to understand long non-coding RNAs. Nature Reviews Genetics 17: 601-614. doi:10.1038/nrg.2016.85

Volders P-J, Verheggen K, Menschaert G, Vandepoele K, Martens L, Vandesompele J, Mestdagh P. 2015. An update on LNCipedia: a database for annotated human lncRNA sequences. Nucleic Acids Res 43 D174-D180. doi:10.1093/nar/gku1060

Xue Z, Hennelly S, Doyle B, Gulati AA, Novikova IV, Sanbonmatsu KY, Boyer LA. 2016. A G-rich motif in the lncRNA Braveheart interacts with a zinc-finger transcription factor to specify the cardiovascular lineage. Mol Cell 64: 37-50. doi:10.1016/j.molcel.2016.08.010

Received July 12, 2019; accepted in revised form June 24, 2020.

\section{Genome Research}




\section{Corrigenda}

Genome Research 28: 285-294 (2018)

\section{Corrigendum: $3^{\prime}$ UTR lengthening as a novel mechanism in regulating cellular senescence} Meng Chen, Guoliang Lyu, Miao Han, Hongbo Nie, Ting Shen, Wei Chen, Yichi Niu, Yifan Song, Xueping Li, Huan Li, Xinyu Chen, Ziyue Wang, Zheng Xia, Wei Li, Xiao-Li Tian, Chen Ding, Jun Gu, Yufang Zheng, Xinhua Liu, Jinfeng Hu, Gang Wei, Wei Tao, and Ting Ni

The authors would like to correct Figure 3, panel J, in which the rightmost upper image of SA- $\beta$-gal stained 293T cells following short hairpin RNA (shRNA)-mediated knockdown of RRAS2 with sh769 (RRAS2-KDsh769) was inadvertently, and due to a labeling error, taken from the same original source image presented in the middle upper panel, which shows increased SA- $\beta$-gal activity following RRAS2 knockdown by a different shRNA (sh646). This correction does not affect any of the conclusions of the article. The corrected image representative of RRAS2-KD-sh769 is provided below, and Figure 3 has been updated in the article online.

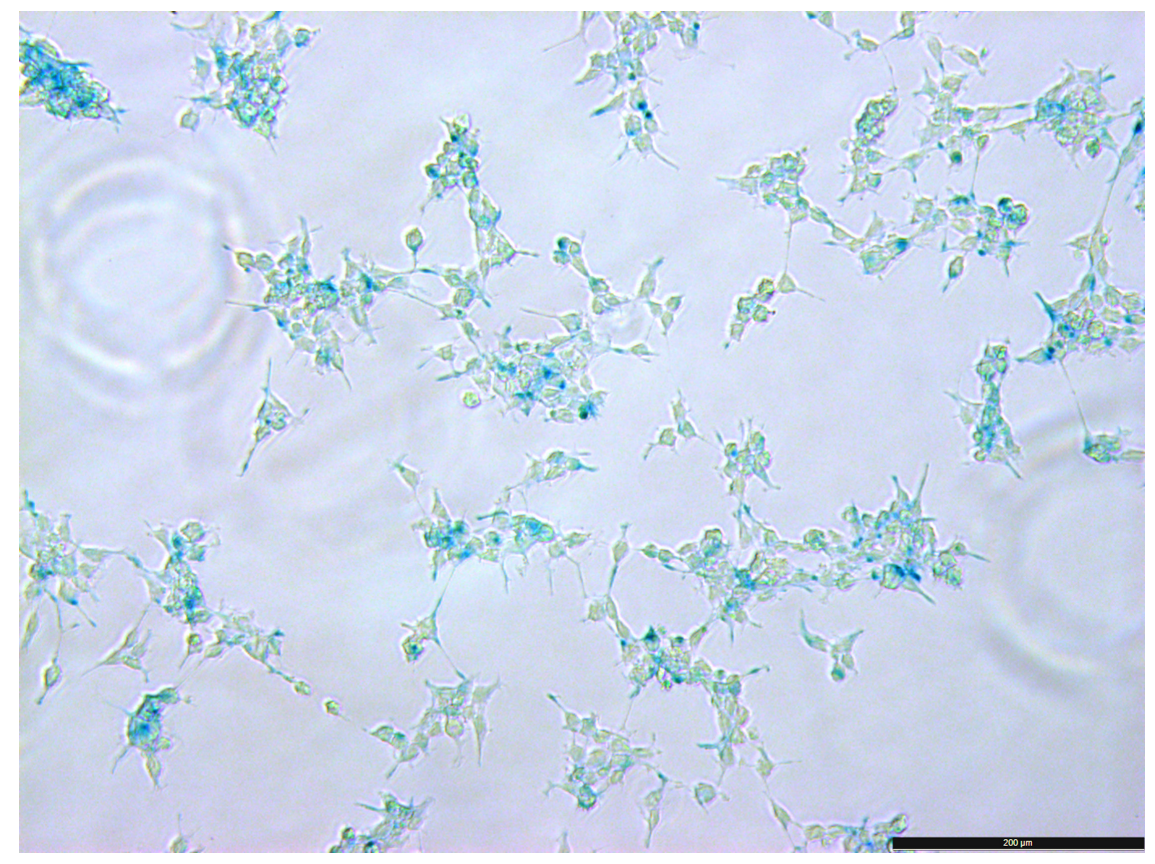

Figure 3. Panel J, rightmost upper image.

The authors thank Ning Yuan Lee for bringing this error to their attention and apologize for any confusion this may have caused.

Additionally, the authors have provided a revised Supplemental Figure S7 file in which the redundant successive Supplemental figure files have been removed. This can be found in the Revised Supplemental Material online.

doi: $10.1101 /$ gr.270165.120 
Genome Research 30: 1060-1072 (2020)

\section{Corrigendum: Functional annotation of human long noncoding RNAs via molecular phenotyping}

Jordan A. Ramilowski, Chi Wai Yip, Saumya Agrawal, Jen-Chien Chang, Yari Ciani, Ivan V. Kulakovskiy, Mickaël Mendez, Jasmine Li Ching Ooi, John F. Ouyang, Nick Parkinson, Andreas Petri, Leonie Roos, Jessica Severin, Kayoko Yasuzawa, Imad Abugessaisa, Altuna Akalin, Ivan V. Antonov, Erik Arner, Alessandro Bonetti, Hidemasa Bono, Beatrice Borsari, Frank Brombacher, Christopher J.F. Cameron, Carlo Vittorio Cannistraci, Ryan Cardenas, Melissa Cardon, Howard Chang, Josée Dostie, Luca Ducoli, Alexander Favorov, Alexandre Fort, Diego Garrido, Noa Gil, Juliette Gimenez, Reto Guler, Lusy Handoko, Jayson Harshbarger, Akira Hasegawa, Yuki Hasegawa, Kosuke Hashimoto, Norihito Hayatsu, Peter Heutink, Tetsuro Hirose, Eddie L. Imada, Masayoshi Itoh, Bogumil Kaczkowski, Aditi Kanhere, Emily Kawabata, Hideya Kawaji, Tsugumi Kawashima, S. Thomas Kelly, Miki Kojima, Naoto Kondo, Haruhiko Koseki, Tsukasa Kouno, Anton Kratz, Mariola Kurowska-Stolarska, Andrew Tae Jun Kwon, Jeffrey Leek, Andreas Lennartsson, Marina Lizio, Fernando López-Redondo, Joachim Luginbühl, Shiori Maeda, Vsevolod J. Makeev, Luigi Marchionni, Yulia A. Medvedeva, Aki Minoda, Ferenc Müller, Manuel Muñoz-Aguirre, Mitsuyoshi Murata, Hiromi Nishiyori, Kazuhiro R. Nitta, Shuhei Noguchi, Yukihiko Noro, Ramil Nurtdinov, Yasushi Okazaki, Valerio Orlando, Denis Paquette, Callum J.C. Parr, Owen J.L. Rackham, Patrizia Rizzu, Diego Fernando Sánchez Martinez, Albin Sandelin, Pillay Sanjana, Colin A.M. Semple, Youtaro Shibayama, Divya M. Sivaraman, Takahiro Suzuki, Suzannah C. Szumowski, Michihira Tagami, Martin S. Taylor, Chikashi Terao, Malte Thodberg, Supat Thongjuea, Vidisha Tripathi, Igor Ulitsky, Roberto Verardo, Ilya E. Vorontsov, Chinatsu Yamamoto, Robert S. Young, J. Kenneth Baillie, Alistair R.R. Forrest, Roderic Guigó, Michael M. Hoffman, Chung Chau Hon, Takeya Kasukawa, Sakari Kauppinen, Juha Kere, Boris Lenhard, Claudio Schneider, Harukazu Suzuki, Ken Yagi, Michiel J.L. de Hoon, Jay W. Shin, and Piero Carninci

The authors would like to correct the misspelling of an author's name and the inadvertent omission of two affiliations for that author, which are as follows: Christopher J.F. Cameron, Department of Biochemistry, Rosalind and Morris Goodman Cancer Research Center, McGill University, Montréal, Québec H3G 1Y6, Canada and Department of Molecular Biophysics and Biochemistry, Yale University, New Haven, Connecticut 06510, USA.

These updates are reflected in the revised manuscript online.

doi: $10.1101 /$ gr.270330.120 


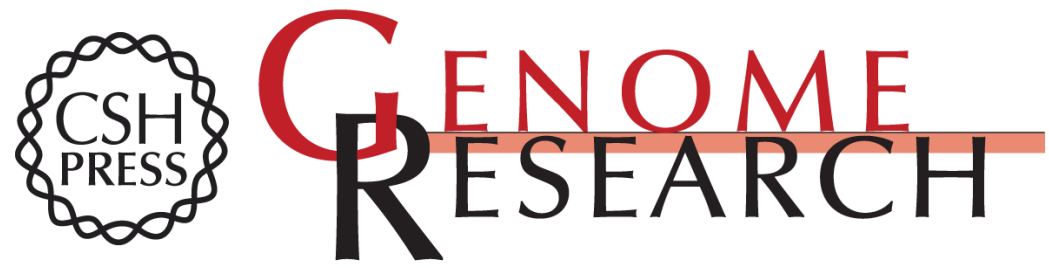

\section{Functional annotation of human long noncoding RNAs via molecular phenotyping}

Jordan A. Ramilowski, Chi Wai Yip, Saumya Agrawal, et al.

Genome Res. 2020 30: 1060-1072 originally published online July 27, 2020

Access the most recent version at doi:10.1101/gr.254219.119

\section{Supplemental http://genome.cshlp.org/content/suppl/2020/07/21/gr.254219.119.DC1 \\ Material}
Related Content Corrigendum: Functional annotation of human long noncoding RNAs via molecular phenotyping
Jordan A. Ramilowski, Chi Wai Yip, Saumya Agrawal, et al.
Genome Res. September , 2020 30: 1377-1

References This article cites 58 articles, 9 of which can be accessed free at:

http://genome.cshlp.org/content/30/7/1060.full.html\#ref-list-1

Articles cited in:

http://genome.cshlp.org/content/30/7/1060.full.html\#related-urls

Open Access Freely available online through the Genome Research Open Access option.

Creative This article, published in Genome Research, is available under a Creative

Commons Commons License (Attribution 4.0 International), as described at

License http://creativecommons.org/licenses/by/4.0/.

Email Alerting Receive free email alerts when new articles cite this article - sign up in the box at the Service top right corner of the article or click here.

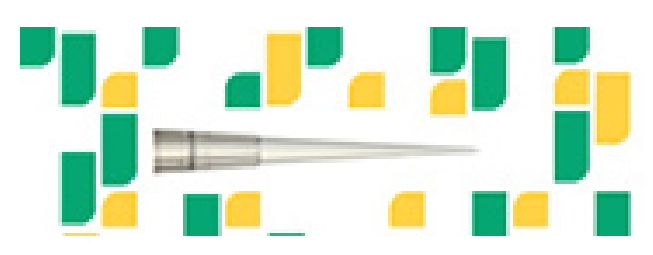

Focused on your science.

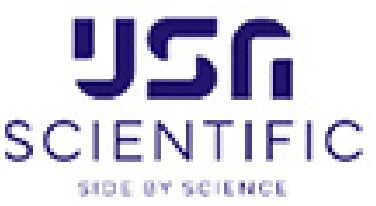

To subscribe to Genome Research go to:

https://genome.cshlp.org/subscriptions

(C) 2020 Ramilowski et al.; Published by Cold Spring Harbor Laboratory Press 